\title{
Determining correlation and coherence lengths in turbulent boundary layer flight data
}

\author{
Dan Palumbo * \\ NASA Langley Research Center, MS 463, Hampton, VA 23681, United States
}

\section{A R T I C L E I N F O}

Article history:

Received 21 March 2011

Received in revised form

8 March 2012

Accepted 13 March 2012

Handling Editor: P. Joseph

Available online 24 April 2012

\begin{abstract}
A B S T R A C T
Wall pressure data acquired during flight tests at several flight conditions are analysed and the correlation and coherence lengths of the data reported. It is found that the correlation and coherence lengths are influenced by the origin of the structure producing the pressure and the frequency bandwidth over which the analyses are performed. It is shown how the frequency bandwidth biases the correlation length and how the convection of the pressure field might reduce the coherence measured between sensors. A convected form of the cross correlation and cross spectrum is introduced to compensate for the effects of convection. Coherence lengths measured in the streamwise direction appear much longer than expected. Coherent structures detected using the convected cross correlation do not exhibit an exponential coherent power decay.
\end{abstract}

Published by Elsevier Ltd.

\section{Introduction}

The correlation length is a measure of the extent of spatially coherent power in the wall pressure and is thus an important parameter in studies of structural response to boundary layer excitation [1-3]. The nature of the boundary layer is particularly important to studies of aircraft interior noise where the boundary layer is the dominant noise source. It has been established, based on data acquired over several years [4,5], that the coherent power in the wall pressure decays exponentially with distance. This behavior is reflected in most models of the wall pressure cross spectrum [6-8]. The models also assume that the wall pressure statistics are homogeneous and stationary [6,9], that is, the wall pressure can be considered to be a single process and statistical quantities such as the power spectrum and cross spectrum are constant over the space/time dimensions of the analyses. Assuming stationarity, the cross spectrum can be derived by scaling the power spectrum according to a coherence length ${ }^{1}$ and adjusting the phase according to the convection wavenumber. This is the basis of the Corcos model [6]. As explained in later sections, the coherence length is similar, but not equivalent, to the correlation length. In the Corcos model, the coherence length is inversely proportional to frequency leading to the unrealistic (in light of available data) prediction that the coherence length in the wall pressure would tend towards infinity as the frequency approached zero. This shortcoming in the Corcos model was addressed by Efimtsov [7] who used boundary layer thickness, $\delta$, friction velocity, $u_{\tau}$, and Strouhal number, $S h$, to fit the coherence length to available data. Several other models have since been proposed (see Graham [11] and Hwang [12] for overviews). The purpose of this study is not to select the best model, but to establish a better understanding of the underlying behavior exhibited by flight data under typical conditions. The Corcos/Efimtsov model will be used to relate the current data and analyses to established norms.

\footnotetext{
*Tel.: +1 757864 6185; fax: +1 7578648823 .

E-mail address: d.l.palumbo@nasa.gov

1 The term 'coherence length' is introduced here to remove ambiguities on how the quantity is calculated and used.
} 
The data that forms the basis for the study were acquired in a flight test that was a cooperative venture between the Gulfstream Aerospace Corporation, Boeing Commercial Airplanes and the National Aeronautics and Space Administration (NASA). The most straightforward way of acquiring pressure data over the surface of an aircraft's fuselage is to flush mount sensors in a window blank. The first comprehensive test was performed by Bhat [5] where longitudinal and lateral arrays of flush mounted sensors were installed in the forward and aft window areas of a Boeing 737. Bhat was able to characterize the wall pressure power spectral density and correlation lengths with both wideband and narrowband analyses. A later test [13] attempted to reduce anomalies in the pressure data that were suspected to stem from the degree of sensor-tosurface flushness. In spite of best efforts, perturbations of amplitude and phase in spectral data were still apparent [14]. The subject data set was acquired using a pinhole array machined in a single piece of stainless steel sheet metal yielding a surface with minimal irregularities. As will be shown, the data are remarkably consistent over the array. The high quality of the data raises confidence in results that show coherence lengths in the streamwise direction several times longer than expected based on previous studies. Values often quoted in the literature for Efimtsov's model's three parameters for streamwise coherence length $\left(a_{1}, a_{2}, a_{3}\right)$ are $0.1,75.4$ and 1.54 [7] and predict a coherence length of $0.1 \mathrm{~m}$ at $1 \mathrm{kHz}$. Analysis of the data reveal a coherence length of $0.45 \mathrm{~m}$ at $1 \mathrm{kHz}$ requiring parameters of $0.06,15$ and 3 . Another recent study also reports a similar trend towards increased measured coherence lengths requiring modifications to model parameters [15]. The observed increased coherence lengths may be due to several factors including improved instrumentation, location of the array, the conditions under which the data were acquired and the processes used to reduce the data. These effects will be discussed in the text. It will be shown that the value of a derived coherent power decay parameter depends on the analysis, i.e., whether it is cross correlation or cross spectrum, and that these analyses can return different results depending on the bandwidth and bin width employed. A convected cross correlation technique is introduced which eliminates the dependence on bin width. The convected cross correlation will be used to detect coherent structures and it will be shown that these structures do not exhibit an exponential power decay in space and that the use of an exponential to characterize the decay results in overestimating the correlation length. The unique behavior of these structures reduces the strength of the homogeneity assumption. In addition, a test for stationarity indicates that the data are weakly stationary, which is adequate for most analyses.

The components of the flight test instrumentation and flight test conditions will be described in the next section. The quality of the data will then be evaluated followed by a discussion on the difference between the streamwise and cross stream results. The data will be put in context of previous flight test data. The theoretical basis for computing the correlation and coherence lengths is established followed by an evaluation of the stationarity of the data and a demonstration of the biased behavior of the cross spectrum when operating on turbulent boundary layer wall pressure data. Explanation of the observed behavior will be provided and the proposed convected forms of the analyses offered as a solution. An example will then be given of how coherent structure is detected in the data and the behavior of the structure explored.

\section{The flight test}

The flight test was conducted as a cooperative effort between the Gulfstream Aerospace Corporation, Boeing Commercial Airplanes and NASA. The Gulfstream aircraft (a G550) was chosen primarily because its large windows provide a good platform for a long, streamwise, array of sensors that could be built into a window blank.

\subsection{The test bed and instrumentation}

The sensor array was located in the first window of the G550,6.7 $\mathrm{m}$ from the nose of the aircraft on the starboard side, Fig 1(a). The array was inclined at an angle of $5.5^{\circ}$ to the fuselage axis to account for the angle of the streamline during cruise, Fig 1(b). The second window was marked with a line in the same relative position as the streamwise array and tufts fitted so that the streamline could be observed and the aircraft made to hold the required angle of attack during acquisition. There were a total of 43 sensors in the streamwise array spanning $47.7 \mathrm{~cm}$ and 15 sensors in the cross stream array spanning $8.1 \mathrm{~cm}$. The sensors in the high density portions of the arrays were spaced 3 mm apart. Additional sensors spaced at $6 \mathrm{~mm}, 12 \mathrm{~mm}$ and $36 \mathrm{~mm}$ were added to increase the length of the arrays beyond the point of expected significant correlation. The pressure sensors were $1.6 \mathrm{~mm}$ in diameter with piezo-resistive elements operated in differential mode, vented to the cabin. These type of sensors have a wide temperature range $\left(-55^{\circ} \mathrm{C}\right.$ to $\left.120{ }^{\circ} \mathrm{C}\right)$ and a wide dynamic range with up upper frequency limit of $150 \mathrm{kHz}$. The sensors have a combined nonlinearity, hysteresis and repeatability of $+/-0.1 \%$ full scale output and a temperature sensitivity of $1 \%$ relative to $100{ }^{\circ} \mathrm{F}$. A pre-installation calibration found the sensors to have sensitivity at $1 \mathrm{kHz}$ of approximately $0.35 \mu \mathrm{V} / \mathrm{Pa}$ and to be within $+/-0.1 \mathrm{~dB}$ of their response at $4 \mathrm{kHz}$ over the band of $0 \mathrm{kHz}$ to $4 \mathrm{kHz}$. The sensors were AC coupled. The pinhole geometry had a diameter of $0.5 \mathrm{~mm}$ and a depth of $0.254 \mathrm{~mm}$, Fig 1 (c). The cavity above the sensors was $1.78 \mathrm{~mm}$ in depth and $1.45 \mathrm{~mm}$ in diameter. The usable bandwidth of the array is limited by the Helmholtz resonance of the cavity and the pinhole dimensions. The Helmholtz resonance frequency for the cavity is approximately $15 \mathrm{kHz}$. At a nominal convection velocity of $150 \mathrm{~m} / \mathrm{s}$ the correction factor for a sensor diameter of $0.5 \mathrm{~mm}$ is less than $5 \%$ at $5 \mathrm{kHz}$ [9]. 
(a)

(b)

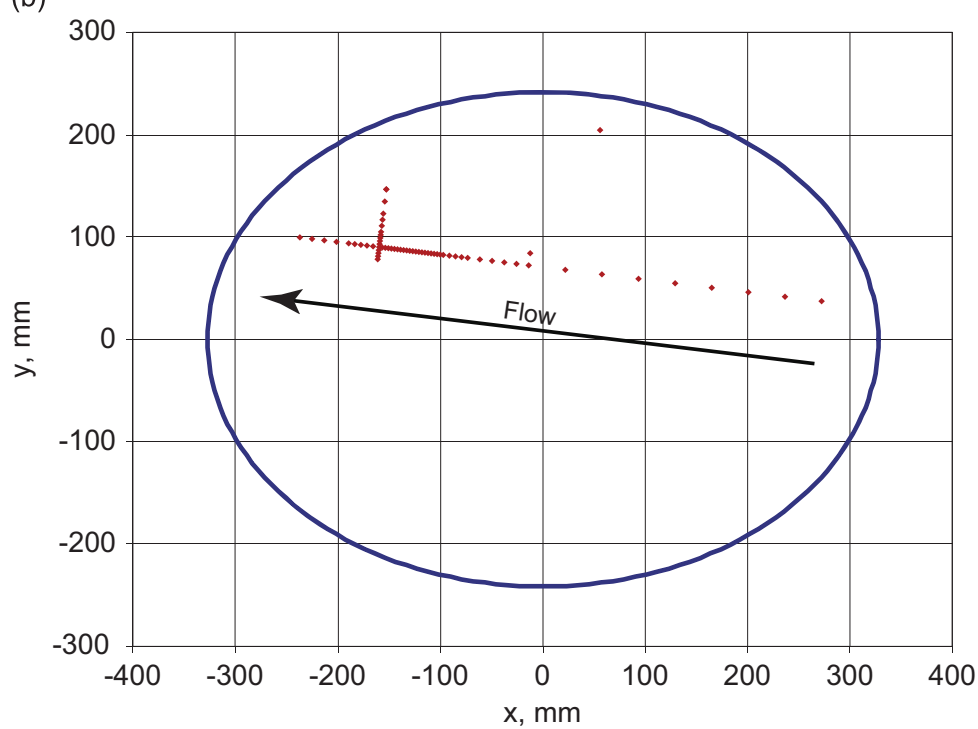

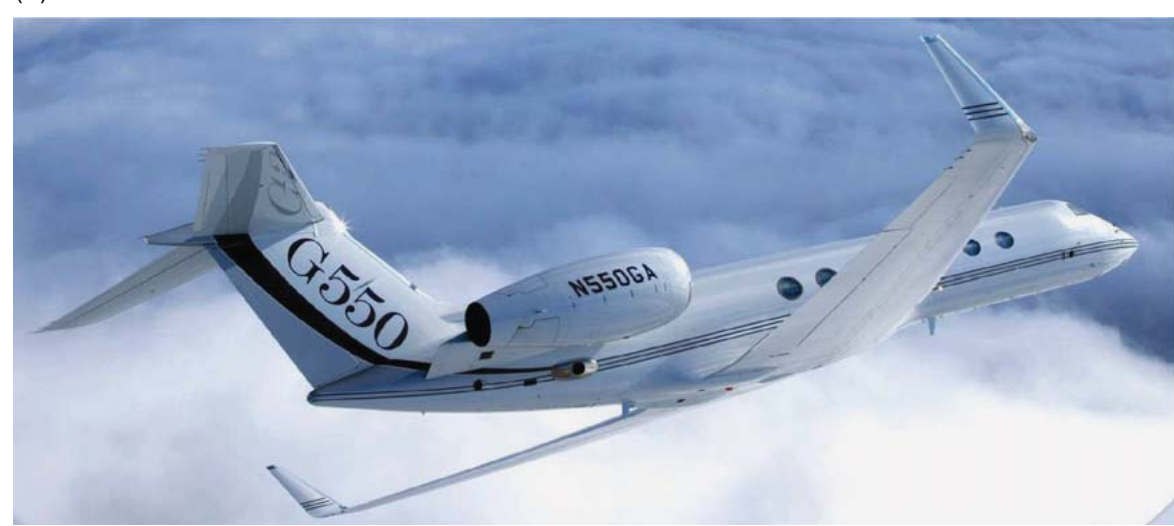

(c)

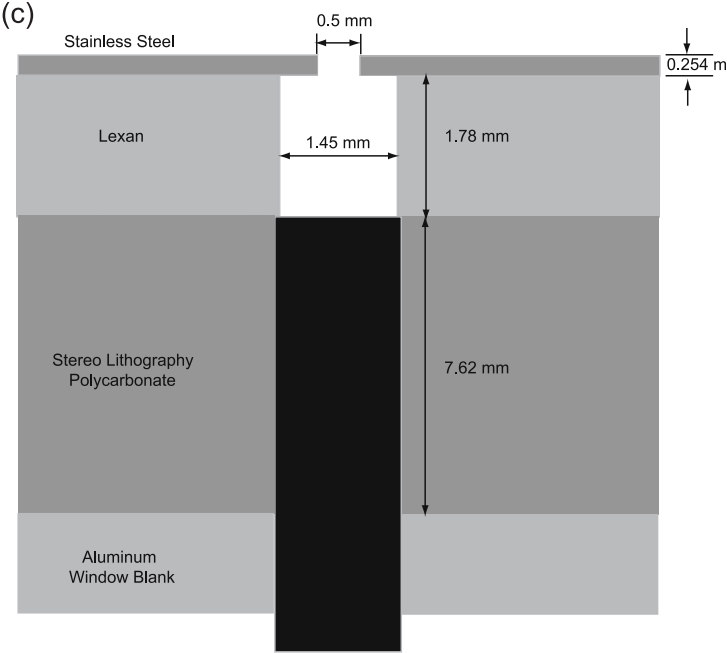

Fig 1. Flight test vehicle (a), position of array in window blank (b), and cross section of pinhole, (c).

\subsection{Signal processing}

The data were acquired at $204.8 \mathrm{kHz}$ to maximize time resolution and for $60 \mathrm{~s}$ to provide adequate time for averaging. The frequency band of primary interest in this study is limited to $3 \mathrm{kHz}$ to avoid the need for correction while still capturing the important behavior. The discrete Fourier transform was performed using the FFTW library [10] subroutines. The transform is taken over $N$ samples in time to produce one ensemble of spectrum. If the data were acquired at a sample rate $f_{s}$, the bandwidth of the spectrum would be $f_{s} / 2$ with $(N+1) / 2$ frequency bins of width $f_{s} / N$. Rectangle windowing with no overlap was employed during averaging. It was found that, over the $3 \mathrm{kHz}$ frequency band of interest, the cross spectra at a separation of $10 \mathrm{~cm}$ and 250 averages had stabilized to within $+/-1 \mathrm{~dB}$ of the cross spectra using 3000 averages (the maximum possible). For computational expedience, 250 averages with a block length of 4096 were used in computing the spectra, unless otherwise noted in the text. The convected cross spectrum (see Section 5.1 on page 25) is computed by shifting the data in the buffers according to sensor separation and estimated convection velocity. The cross correlation is taken as the inverse transform of the cross spectrum.

\subsection{Flight test conditions}

Data were acquired at 3 flight conditions as summarized in Table 1 where $c$ is the speed of sound, $U_{\infty}$ is the free stream velocity, $x$ is the distance from the nose to the window blank, $v$ is the kinematic viscosity, Re is the Reynolds number based on $x, C_{f}$ is the coefficient of skin friction, $u_{\tau}$ is the friction velocity and $\delta$ is the boundary layer thickness. The aircraft was held at constant Mach number and angle of attack during data acquisition. The following relationships were used to derive the quantities shown in Table 1 given the altitude and Mach number taken from the aircraft's air data computer and the distance from the nose to the window blank provided by the manufacturer. The speed of sound in air, $c$, and the kinematic viscosity, $v$, are taken from the Aerospaceweb's Atmospheric Properties 
Table 1

Flight test conditions and flow parameters.

\begin{tabular}{|c|c|c|c|c|c|c|c|c|c|c|}
\hline Flight number & Mach number & Altitude (ft) & $c(\mathrm{~m} / \mathrm{s})$ & $U_{\propto}(\mathrm{m} / \mathrm{s})$ & $x(\mathrm{~m})$ & $v(\mathrm{~m} 2 / \mathrm{s})$ & $R e$ & $C f$ & $u_{\tau}(\mathrm{m} / \mathrm{s})$ & $\delta(\mathrm{m})$ \\
\hline B101 & 0.86 & 48,000 & 295 & 252 & 6.7 & $6.9 E-5$ & 2.5E7 & $2.0 E-3$ & 7.9 & $8.5 E-2$ \\
\hline B106 & 0.7 & 32,000 & 301 & 210 & 6.7 & $3.4 E-5$ & 4.1E7 & $1.8 \mathrm{E}-3$ & 6.3 & $7.7 E-2$ \\
\hline B109 & 0.56 & 24,000 & 311 & 174 & 6.7 & $2.7 E-5$ & 4.3E7 & $1.8 \mathrm{E}-3$ & 5.2 & $7.6 \mathrm{E}-2$ \\
\hline
\end{tabular}

Calculator $^{2}$ which is based on US Standard Atmosphere 1976.

$$
\begin{gathered}
U_{\infty}=M c \\
R e=x U_{\infty} / v \\
\delta=0.382 x R e^{-0.2} \\
C_{f}=0.0594 R e^{-0.2} \\
u_{\tau}=\left(0.5 U_{\infty}^{2} C_{f}\right)^{0.5}
\end{gathered}
$$

\section{The data}

Before evaluating the data, it is instructive to review characterizations of wall pressure spectra that have formed over the years. These are summarized by Hwang [12] based on observations by Blake [16], Farabee [17] and Bull [4] among others. The expected shape of the wall pressure power spectrum is based on a determination of which scaling variables work best in a particular frequency range. Of note is the division of the spectrum into 4 sections, Fig 2 . The low frequency (up to $\left.\omega \delta / u_{\tau}=5\right)$ and mid-frequency $\left(\omega \delta / u_{\tau}<100\right)$ sections are dominated by structures in the outer layer. The high frequencies $\left(\omega v / u_{\tau}^{2}>0.3\right)$ are dominated by structures in the inner layer. The center section exhibits behavior characteristic of the inner and outer layers. In a study by Hutchins [18], several different data sets are analysed to demonstrate the existence of very long structures in the log region and their influence on inner wall dynamics. Hutchins refers to a "footprint" impressed by structures in the log region on the inner wall. The wall pressure spectrum in the low and mid frequency ranges are then composed of pressure fluctuations originating in physical processes that occur both near the wall and away from the wall. This duality contributes to a non-homogeneous behavior that will be discussed in later sections. Using the flow parameters in Table 1 and frequency in Hertz, $(\mathrm{Hz})$, the power spectrum level is expected to increase with a slope proportional to $f^{2}$ in the low frequency region, up to $65 \mathrm{~Hz}$. The level is then expected to continue to increase in the mid frequencies until it reaches a peak at about $650 \mathrm{~Hz}$. The level than gradually decreases until it enters the center frequency range at $1.3 \mathrm{kHz}$ where the slope becomes proportional to approximately $f^{-1}$. The inner scale region is reached at $55 \mathrm{kHz}$ above which the slope becomes proportional to $f^{-5}$. A well behaved data set should exhibit these characteristic behaviors. The power spectrum for the sensor at the intersection of the streamwise and cross stream arrays for the $0.7 \mathrm{M}$ case is shown in Fig 2 across the full bandwidth. The frequencies mentioned above are marked and the expected slopes indicated. The spectrum exhibits the predicted behavior except for the low frequency region (below $65 \mathrm{~Hz}$ ) where the spectrum does not begin to roll off with a slope proportional to $f^{2}$ until $20 \mathrm{~Hz}$. The spectrum below the peak (at about $1 \mathrm{kHz}$ ) appears to decay with a slope closely proportional to $f^{0.3}$. This is in reasonable agreement with the $f^{0.2}$ slope observed by Leclercq in this region [19]. An anticipated defect occurs above $10 \mathrm{kHz}$ where the effect of the Helmholtz cavity resonance perturbs the spectrum. The true behavior of the spectrum in this region cannot be known, but the shape of the spectrum outside the influence of the cavity resonance appears to be as expected.

\subsection{Data consistency}

A subset of sensors in both the streamwise and cross stream arrays were sampled and the power spectra and cross spectra compared at each frequency. The cross spectra were taken for pairs of sensors spaced at $3.6 \mathrm{~cm}$ for the streamwise array and $0.9 \mathrm{~cm}$ for the cross stream array. The distributions of the difference in the power spectra between the sensors in the streamwise array over a band from $100 \mathrm{~Hz}$ to $3 \mathrm{kHz}$ are shown in Fig 3 for the $0.7 \mathrm{M}$ case. This result is representative of the other 2 flight conditions. For all cases sampled in the streamwise array, $90 \%$ of the differences between the power spectra were less than $+/-1 \mathrm{~dB}$ and $95 \%$ of the differences between the cross spectra were less than $+/-1 \mathrm{~dB}$.

The same analysis (except for the cross spectrum being taken at $0.9 \mathrm{~cm}$ ) for the cross stream array produced less consistent results, Fig 4 . The $0.7 \mathrm{M}$ and $0.56 \mathrm{M}$ cases exhibited similar behavior with $45 \%$ of the power spectrum differences below $1 \mathrm{~dB}$ and $35 \%$ of the cross spectrum differences below $1 \mathrm{~dB}$. The $0.86 \mathrm{M}$ case had much tighter

\footnotetext{
${ }^{2}$ http://www.aerospaceweb.org/design/scripts/atmosphere
} 


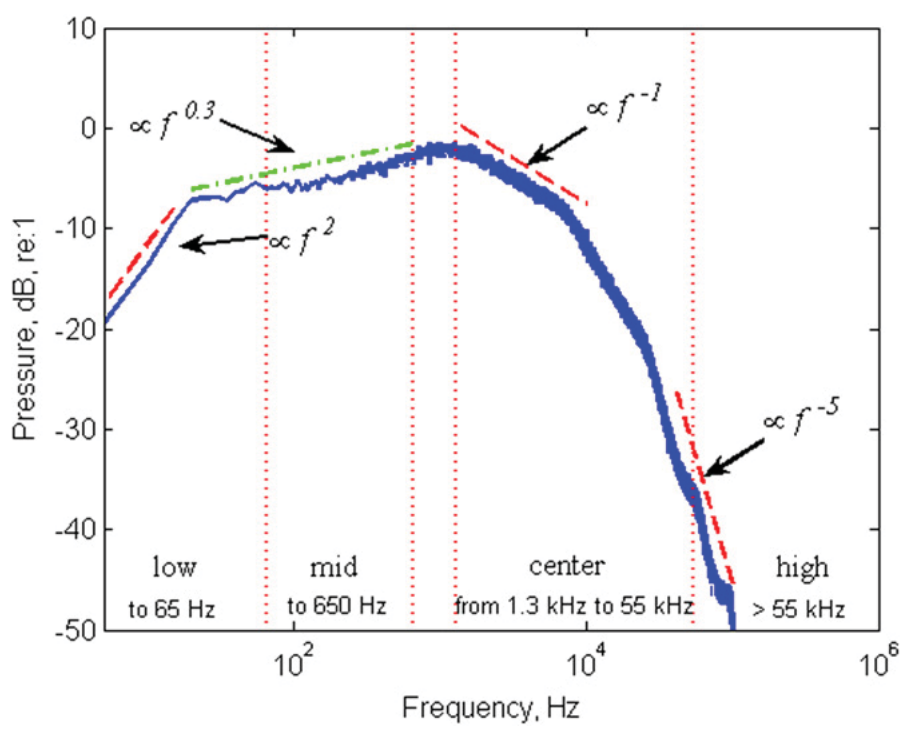

Fig 2. Power spectrum of a typical sensor at $0.7 \mathrm{M}$; __ expected slope; _-_-. estimated slope. (For interpretation of the references to colour in this figure legend, the reader is referred to the web version of this article.)
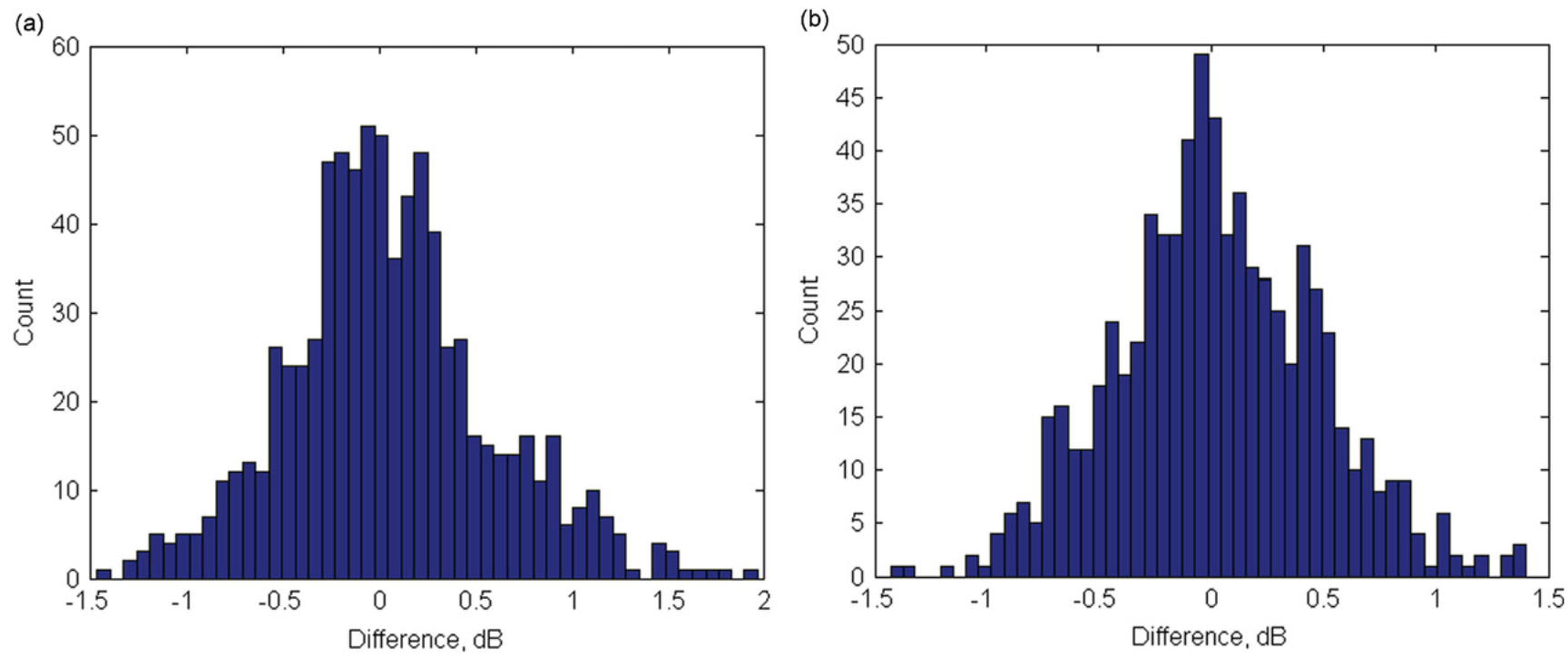

Fig 3. Differences in power spectrum (a), and cross spectrum at $3.6 \mathrm{~cm}$ (b), for sensors in streamwise array at $0.7 \mathrm{M}$.

distributions with $70 \%$ of the power spectrum differences below $1 \mathrm{~dB}$, and $65 \%$ of the cross spectrum differences below $1 \mathrm{~dB}$.

The variation in power over the cross stream array is better understood by viewing the individual power spectra. The power spectra for the sensors used to obtain the power differences for the cross stream array are shown in Fig 5 for the $0.7 \mathrm{M}$ case (a) and the $0.86 \mathrm{M}$ case (b). The direction of increasing $y$ dimension is indicated. As can be seen, the levels of both spectra decrease with increasing $y$. For the $0.7 \mathrm{M}$ case (and the $0.56 \mathrm{M}$ case) the overall reduction in level is accompanied by a gradual elimination of the peak at $1 \mathrm{kHz}$. The power at $1 \mathrm{kHz}$ decreases by nearly $5 \mathrm{~dB}$ over the $8.1 \mathrm{~cm}$ length of the array at $0.7 \mathrm{M}$. The spectra at $0.86 \mathrm{M}$ retains its shape, but the peak is shifted slightly to lower frequencies, decreasing just over $2 \mathrm{~dB}$. The tighter grouping and retention of the spectra's basic shape explains why the power differences for the $0.86 \mathrm{M}$ case are less than the $0.7 \mathrm{M}$ and $0.56 \mathrm{M}$ cases. The flattening of the low speed pressure curves with increasing $y$ may indicate a reduction in log region influence on the wall pressure that appears to persist at the $0.86 \mathrm{M}$ case. The change in spectral pressure over the span of the cross stream array might be due to a pressure gradient caused by the curvature of the aircraft fuselage. Under these conditions, the log region begins about $0.5 \mathrm{~mm}$ (100 wall units) from the wall. If the structures in the log region do impress a footprint on the wall pressure as suggested by Hutchins [18], the slightest modulation of the local pressure could result in a large variation in the impressions. Just such a mechanism is described by Bhat [5] as a possible explanation for the higher correlation decay rates he observed. The difference in behavior between the low speed $(0.7 \mathrm{M}$ and $0.56 \mathrm{M})$ cases and the high speed, $0.86 \mathrm{M}$, case may be due to a slight 
(a)

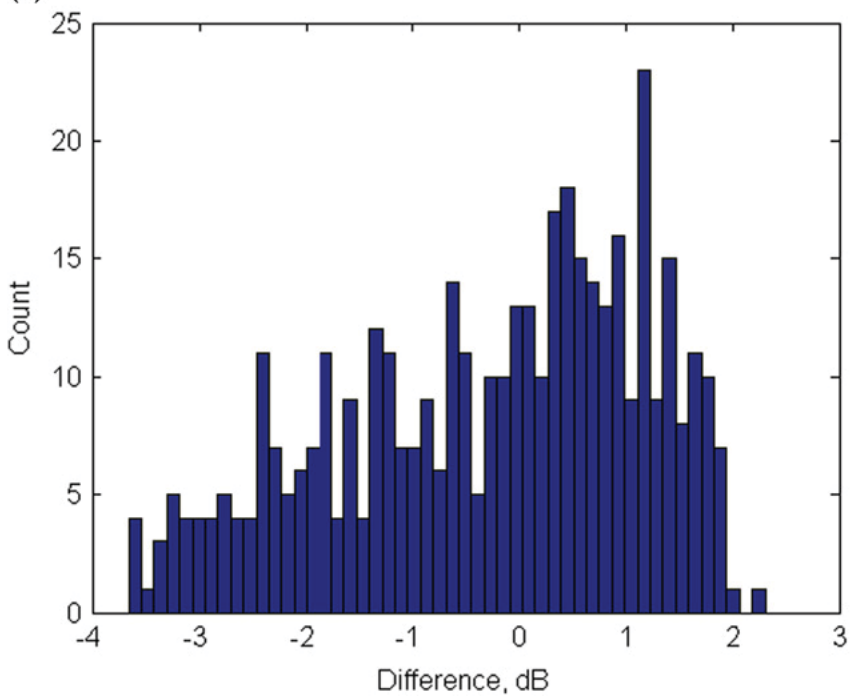

(b)

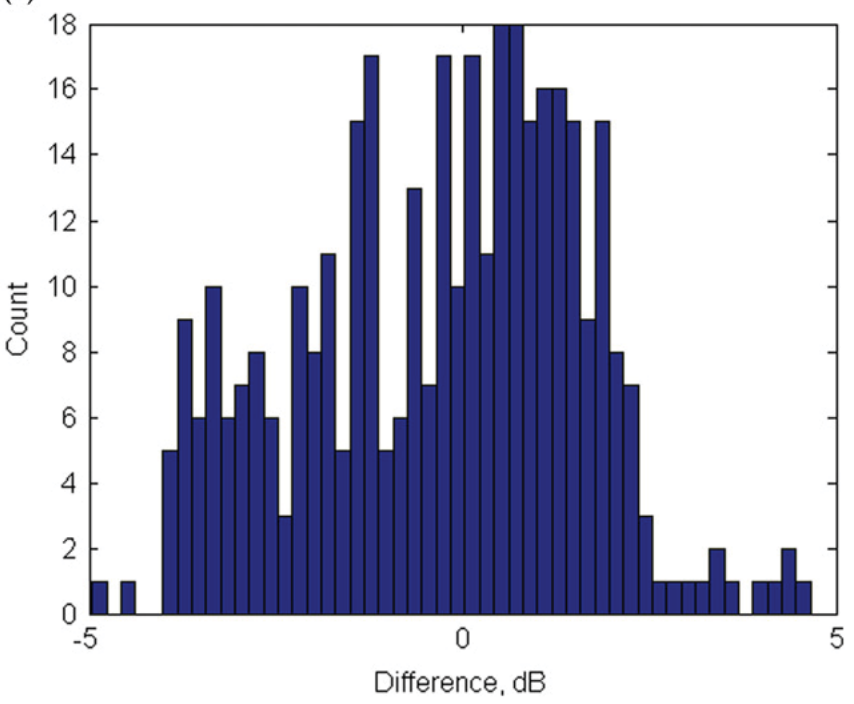

Fig 4. Differences in power spectrum (a), and cross spectrum at $0.9 \mathrm{~cm}$ (b), for sensors in cross stream array at $0.7 \mathrm{M}$.

(a)

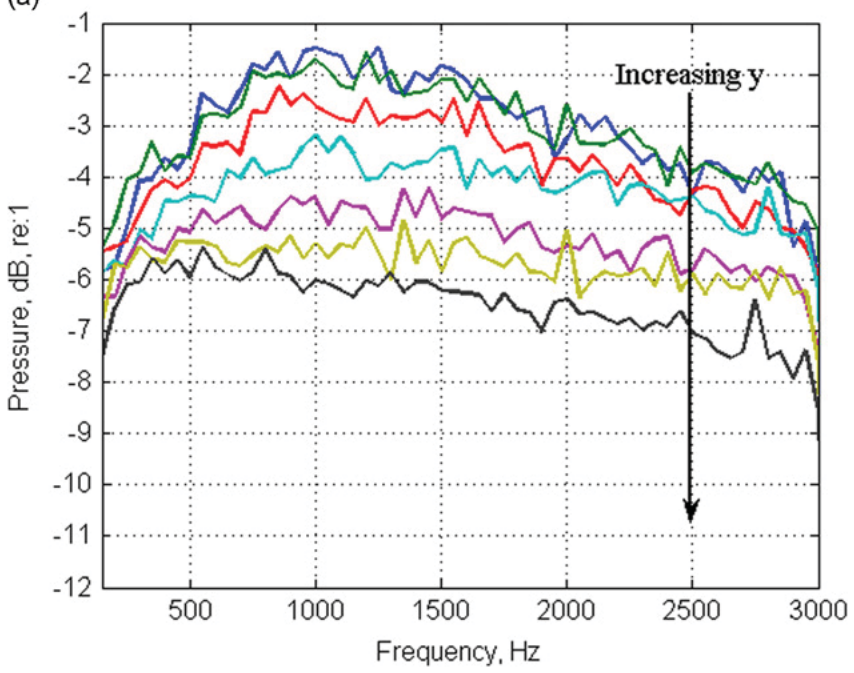

(b)

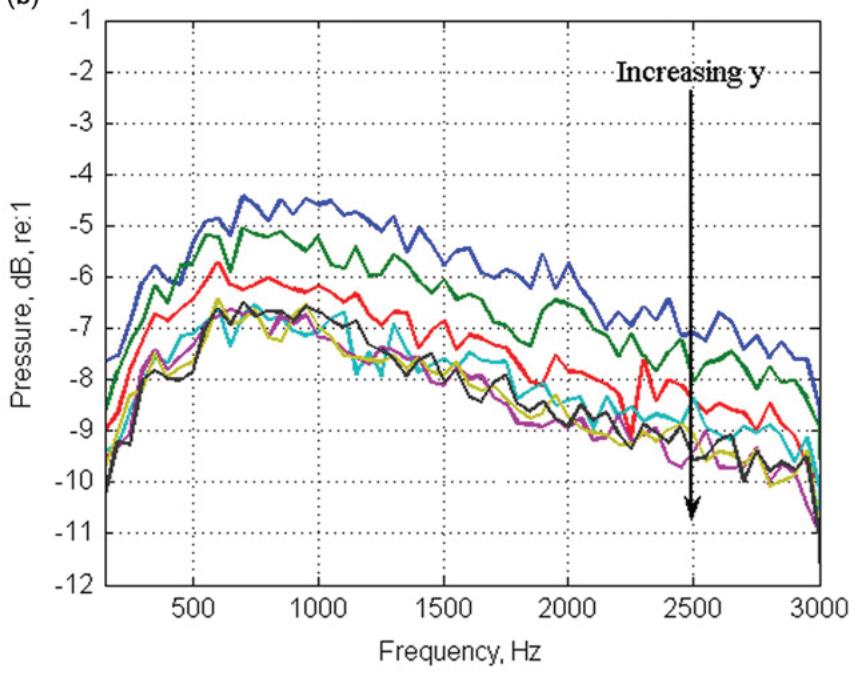

Fig 5. Evolution of power spectra over cross stream array for $0.7 \mathrm{M}$ (a), and $0.86 \mathrm{M}$ (b).

difference in angle of attack or the difference in Reynolds number (from Table 1, the Reynolds number for the $0.86 \mathrm{M}$ case is $35 \%$ below the lower speed cases).

\subsection{Confidence intervals}

The primary measurement is that of the instantaneous pressure. The mean of this value is artificially forced to zero as the data were acquired AC coupled. Better measures, and ones more related to the subject matter of this paper, are the mean square power and the mean cross power. One sample of the mean power is taken from 4096 pressure measurements. This length ensemble was chosen because it was the smallest length at which the effects of convection are diminished (as will be explained in the following sections). Using this ensemble length, it is possible to take 3000 samples of the mean power. The large number of samples guarantees a normal distribution according to the Central Limit Theorem. This is shown to be the case for the mean square power in Fig 6(a). The mean cross power was similarly distributed. The confidence interval for a normally distributed random variable with unknown standard deviation is [20]

$$
\operatorname{ci}(\alpha, n, s)= \pm t_{1-(\alpha / 2), n-1}\left(\frac{s}{\sqrt{n}}\right)
$$

where $c i$ is the confidence interval, $\alpha$ is the complement of the desired probability that the measurement is in the interval, $t$ is the student's $t$ distribution, $n$ is the number of samples and $s$ is the standard deviation of the sample set. Using $n=3000$, 
(a)

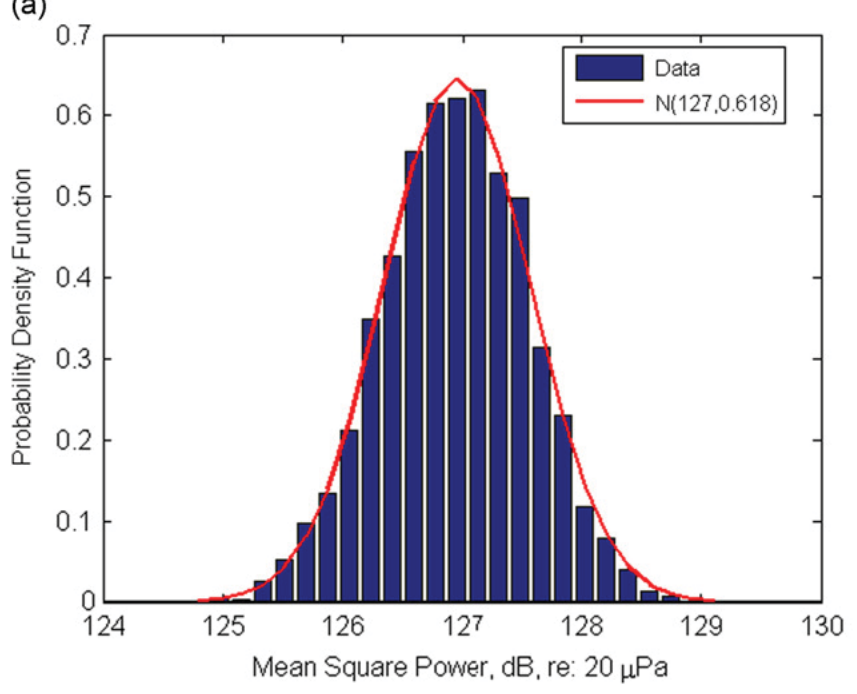

(b)

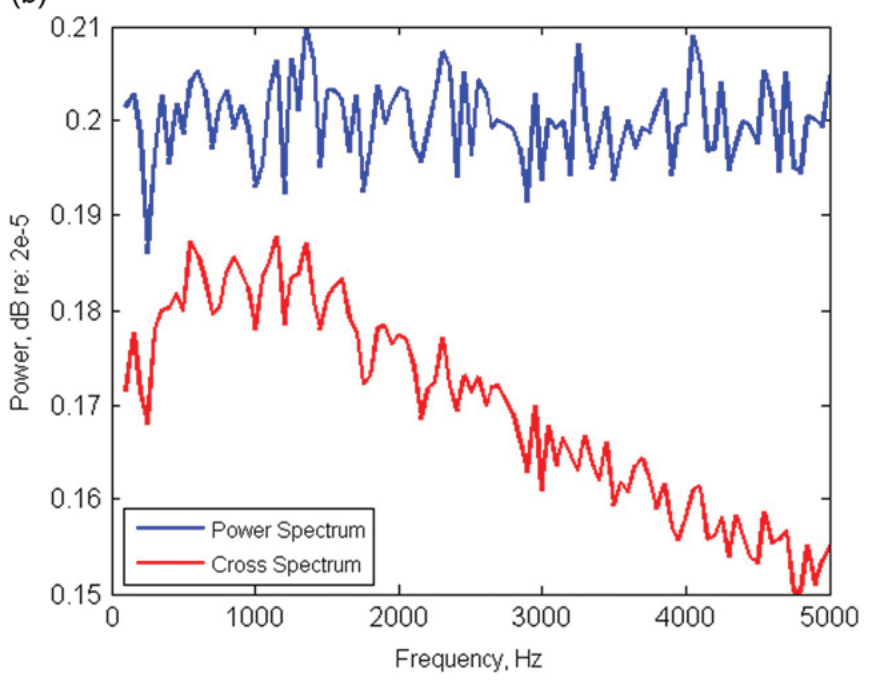

Fig 6. Distribution of samples of mean square power (a), confidence intervals in frequency domain (b).

(a)

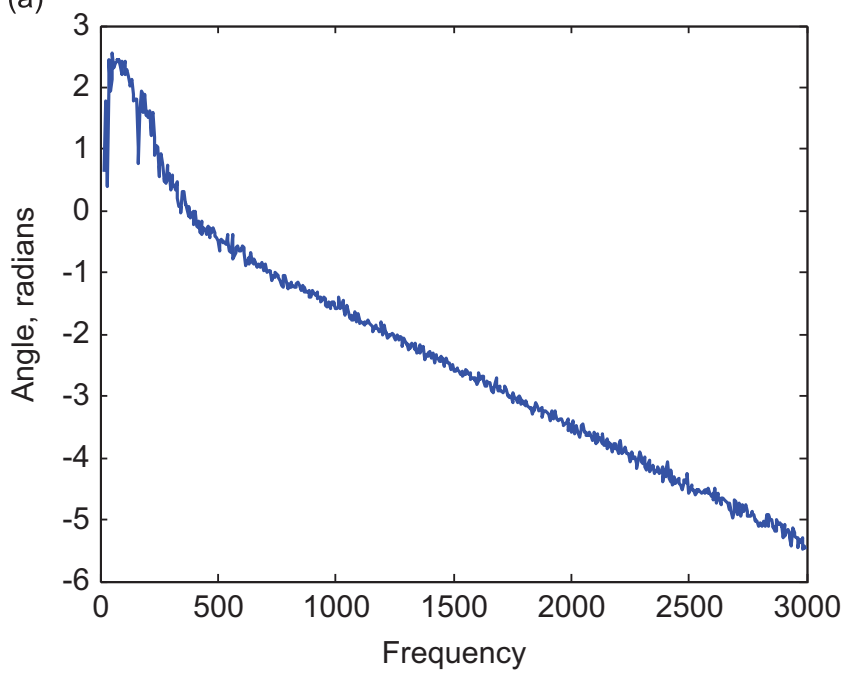

(b)

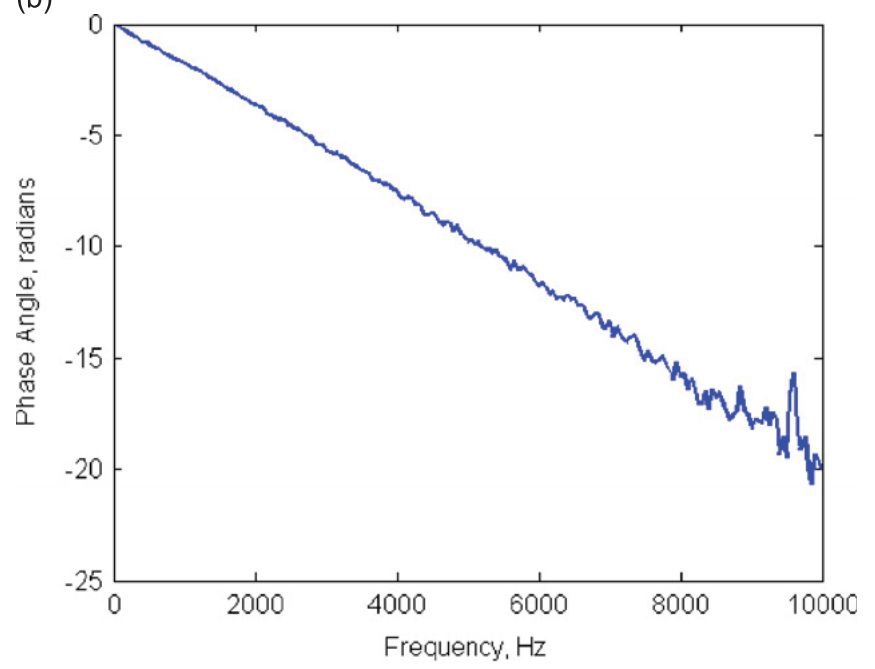

Fig 7. Cross spectrum phase angle between 2 sensors, flush mounted sensors (a), and pinhole sensors (b).

$s=0.618$ and $\alpha=0.01$, the analysis was run for several sensor pairs with spacings varying from $5 \mathrm{~cm}$ to $45 \mathrm{~cm}$. The results for the mean square power were very consistent across the array with typical values shown in Fig 6(a). The mean square power was found to be $127 \mathrm{~dB}$ with a $99 \%$ confidence interval of $0.022 \mathrm{~dB}$. The mean cross power is found to be $113 \mathrm{~dB}$ at separations above $10 \mathrm{~cm}$. with a $99 \%$ confidence interval of $0.17 \mathrm{~dB}$. A similar analysis was done in the frequency domain with the results shown in Fig 6(b). The frequency domain confidence intervals for the mean square power have increased an order of magnitude over the time domain values, but are still a fraction of a dB. With fewer averages the confidence intervals will increase, becoming 3 times larger with 250 averages.

\subsection{Comparison to previous flight data}

In a previous flight test [21] it was noted that flush mounted sensors, despite best efforts to achieve flushness, could effect sensor power spectrum and phase angle. The pinhole array has eliminated those effects, but exposed others. Fig 7(a) illustrates the type of phase error that could occur with flush mounted sensors. The phase behavior of the pinhole data is quite good from very low frequencies up to $10 \mathrm{kHz}$ where the effect of the Helmholtz resonance begins to perturb the phase, Fig 7(b).

Sensor to sensor and flight to flight variation in power spectrum had been attributed to the lack of sensor flushness in previous tests. The consistency of the power spectrum as measured by the pinhole array along the streamwise direction leads one to believe that the variation in the power spectrum along the cross stream array must then be attributed to changing behavior in the boundary layer. The range of variation in the power spectrum measured with the pinhole array is 
(a)

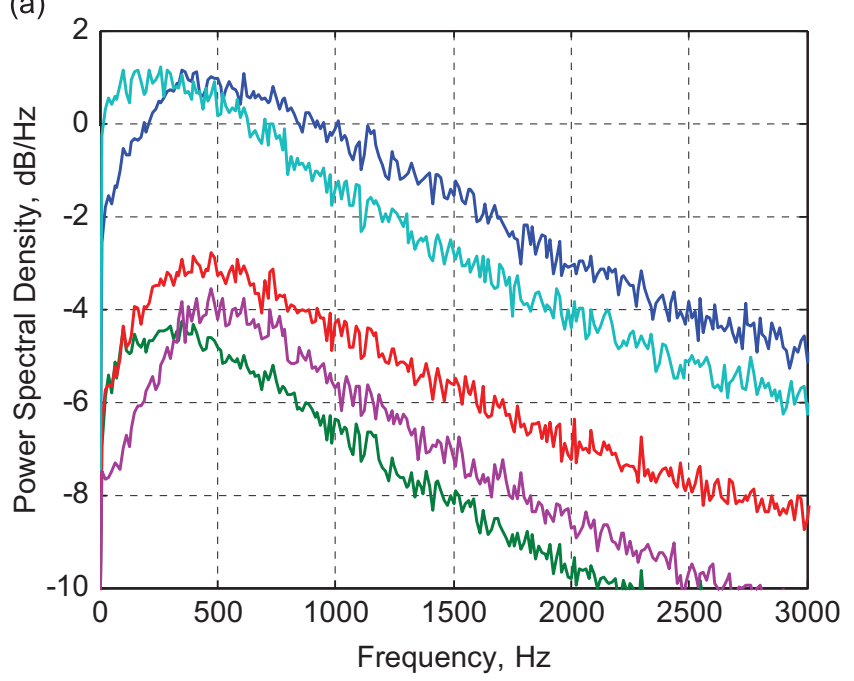

(b)

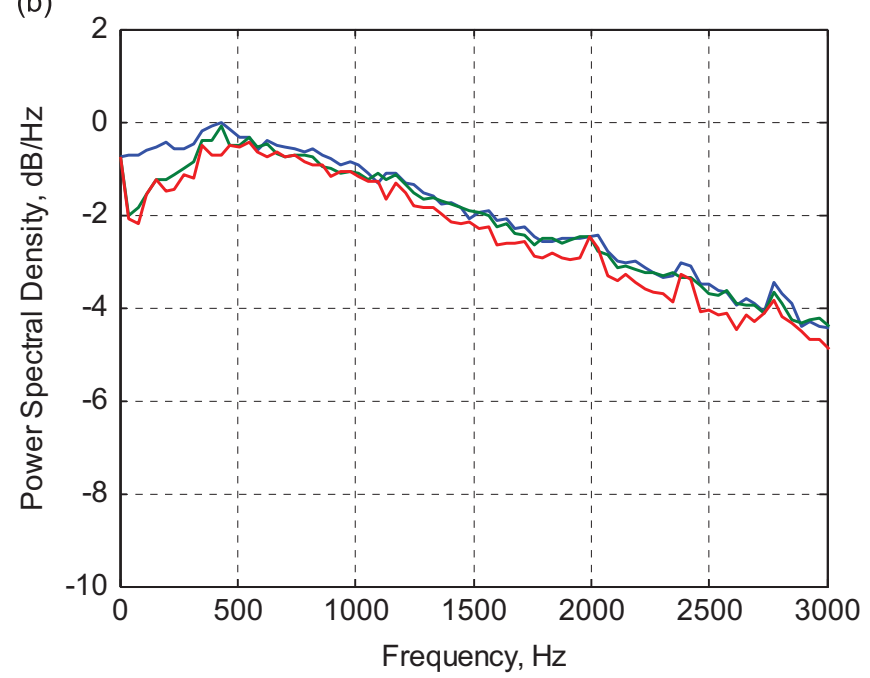

Fig 8. Power spectrum from flush mounted sensors, MD-90 (a), Tu-144 (b).

similar in nature to the changes in the power spectrum observed in previous flight test data. Power spectra from two flight tests are shown in Fig 8. The data are from an MD-90 [22] (a) and a Tu-144 [13] (b). Both tests used different flush mounted sensors. Condenser microphones were used on the MD-90 and piezo-resistive sensors were used on the Tu-144. Although the flights were at different Mach numbers (0.8 M for MD-90 and 0.6 M for Tu-144) the location of the sensors were such that the Reynolds numbers were reasonably close, approximately $1.5 \times 10^{8}$. It can be seen that for some locations and, under some conditions, the power spectrum has a pronounced peak similar to that observed in the streamwise pinhole array. The presence of the peak in the previous tests cannot be attributed to location alone as the data from a particular sensor may or may not have the peak depending on the flight condition. The similarity in the shape of the spectra of the flush mounted sensors to the pinhole array under very different conditions and at different locations on the aircraft reduces the likelihood that the peaks in the spectra were due solely to lack of sensor flushness. It is more likely that this behavior is representative of the variation in physical environment that can occur over the surface of the aircraft. This conclusion weakens the assumption that a single boundary model is valid over the surface of the aircraft fuselage.

\section{Correlation and coherence length analyses}

The term 'correlation length' is often used to describe coherent power decay rates derived by both the cross correlation and the cross spectrum. The resulting rates are quite different, however, and using the same term for both quantities introduces ambiguity. In this study, the term correlation length will be used to describe coherent power decay rates computed using the cross correlation and 'coherence length' for rates computed using the cross spectrum. The space-time cross correlation in one spatial dimension is written as

$$
\chi\left(x_{0}, x_{1}, \tau\right)=\left\langle p\left(x_{0}, t_{0}\right) p\left(x_{1}, t_{0}+\tau\right)\right\rangle
$$

Here $p(x, t)$ is the pressure at some point in space and time and the brackets, $\langle\cdots\rangle$, denote the average over $N$ samples in time. The coherent power decay rate is determined from cross correlation analysis by fitting an exponential distribution to the peaks in the cross correlation taken from a reference sensor to other sensors in the array, see Eq. (8) and Fig 9(a).

$$
\frac{\max _{\tau}\left(\chi\left(x_{0}, x_{i}, \tau\right)\right)}{\left\langle p\left(x_{0}, t\right)^{2}\right\rangle^{1 / 2}\left\langle p\left(x_{i}, t\right)^{2}\right\rangle^{1 / 2}} \cong e^{-\frac{\left|x_{0}-x_{i}\right|}{L}}
$$

where function $\max _{\tau}$ returns the maximum value of $\chi$ for sensor pair, $\left(x_{0}, x_{i}\right)$ over the range of delays, $\tau$. The cross correlation is normalized to the RMS power in each sensor so that the peak in the auto correlation is 1 . The correlation length, $L$, is determined by the value that produces the best fit over all the normalized correlation peaks.

The space-time correlation is related to the cross spectrum by the Fourier transform.

$$
\phi\left(x_{0}, x_{1}, f\right)=\int_{N} \chi\left(x_{0}, x_{1}, \tau\right) e^{-i 2 \pi f \tau} \mathrm{d} \tau
$$

The cross spectrum magnitudes are normalized by the square root of the respective auto spectra so that the resulting quantity is equivalent to the square root of the coherence.

$$
\gamma\left(x_{0}, x_{1}, f\right)=\frac{\left|\phi\left(x_{0}, x_{1}, f\right)\right|}{\left|\phi\left(x_{0}, x_{0}, t_{0} f\right)\right|^{0.5}\left|\phi\left(x_{1}, x_{1}, f\right)\right|^{0.5}}
$$


(a)

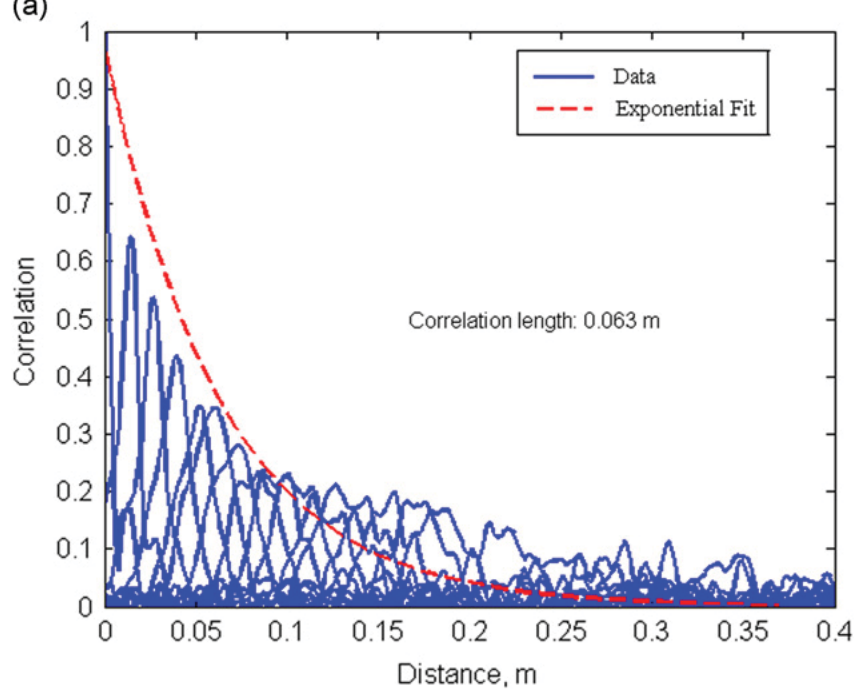

(b)

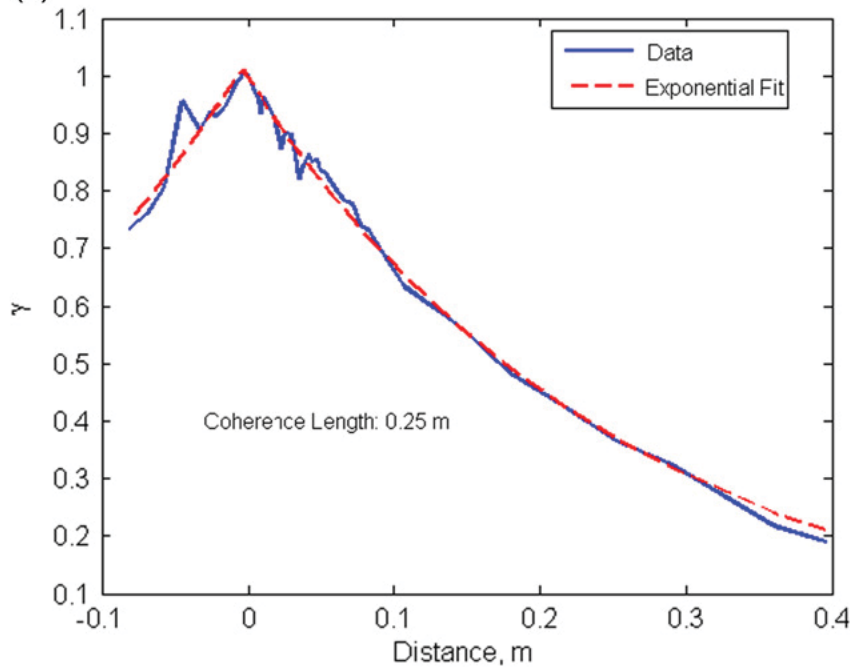

Fig 9. Correlation length determined by exponential fit to peaks in cross correlation (a), and coherence length determined by exponential fit to cross spectrum magnitude at $1500 \mathrm{~Hz}$, (b).

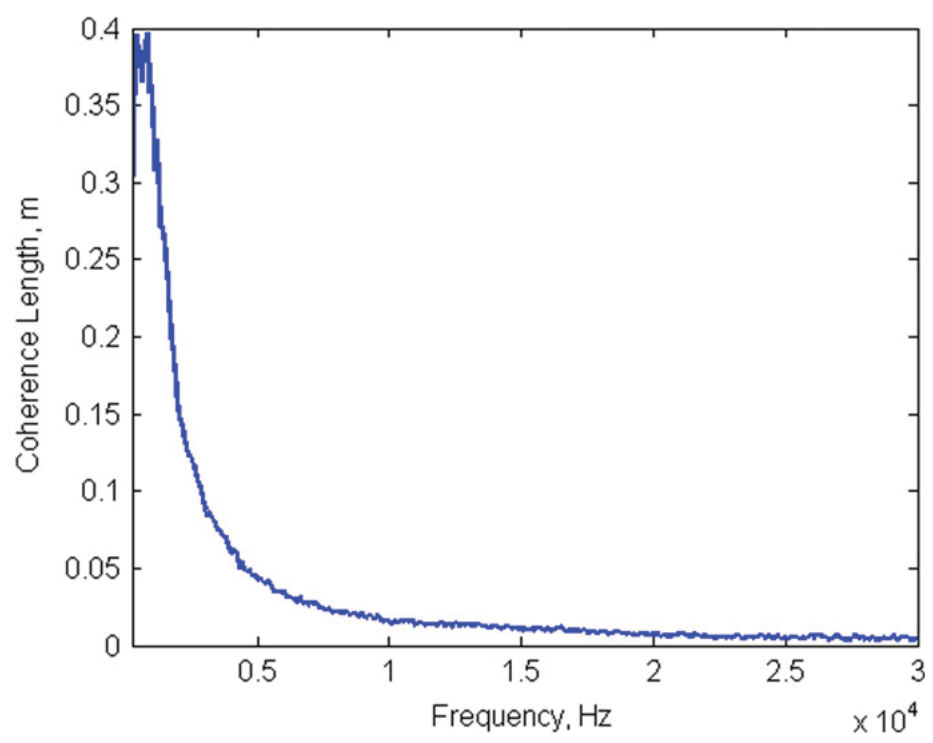

Fig 10. Coherence length up to $30 \mathrm{kHz}$.

The coherence length for a particular frequency is found by fitting an exponential to $\gamma$ at that frequency.

$$
\gamma\left(x_{0}, x_{1}, f_{j}\right) \cong e^{-\frac{\left|x_{0}-x_{i}\right|}{L_{j}}}
$$

An example of an exponential fit to $\gamma$ in the streamwise direction at $1.5 \mathrm{kHz}$ is shown in Fig 9(b). Two things are immediately obvious when comparing the coherence length in Fig 9(b) to the correlation length in Fig 9(a). First, the coherence has a much better fit to the exponential than the cross correlation. This is true at all frequencies. Second, the coherence length at this frequency is much longer than the correlation length. Both these observations can be understood by considering the plot in Fig 10 of the coherence length over the band from $100 \mathrm{~Hz}$ to $30 \mathrm{kHz}$.

The coherence length is strongly frequency dependent reaching its maximum at frequencies corresponding to the peak in the power spectrum, i.e., $\sim 1 \mathrm{kHz}$, and decreasing rapidly at higher frequencies. At high frequencies, structures in the inner layer which decay rapidly dominate the wall pressure behavior. At low frequencies, structures in the outer layer dominate with much longer decay lengths. The cross correlation is a wide band analysis in that its result is a combination of these behaviors over all the frequencies. At short distances the short decay lengths of the structures at the higher frequencies cause a rapid fall off in correlated power and at greater distances, the longer decay lengths of the low frequency structures sustain the correlated power. The lack of consistency in the correlation decay processes across the frequency band results in a behavior that cannot be adequately modeled by a single exponential curve. This is the reason for the less than optimal fit in Fig 9(a) at greater distances. 
The disparity in behavior can be reduced by narrowing the analysis frequency band to a region of interest, for example, around the $1 \mathrm{kHz}$ peak in the power spectrum. The results of deriving the correlation and coherence length from data that was band limited to $100 \mathrm{~Hz}$ to $3 \mathrm{kHz}$ are shown in Fig 11. The coherence length is, of course, unchanged. The correlation length, however, has increased over 350\% illustrating the effect of removing most of the high frequency, inner layer, behavior. The correlation length will approach the coherence length as the frequency band is narrowed and the variety of physical processes at work reduced. At this point, one might conclude that the cross spectrum is the more reliable statistic. That is not entirely the case as will be demonstrated in the following sections.

\subsection{A measure of stationarity}

The use of the averaged cross spectrum assumes the data are stationary, that is, the statistics are constant regardless of the time the data are taken. To be strictly stationary, all moments of the data must be shown to be constant [23]. To reject the assumption of stationarity, it is sufficient to show that the joint probability density function, $f(x, y)$, varies appreciably over the interval.

$$
P(r<X<s, t<Y<u)=\int_{r}^{s} \int_{t}^{u} f(x, y) \mathrm{d} y \mathrm{~d} x
$$

In Eq. (12), $X$ and $Y$ are statistics taken from two samples of data taken at different times from the same channel and $P$ is the probability that $X$ is between $(r, s)$ and $Y$ is between $(t, u)$. The constancy of $f(x, y)$ is tested by dividing the dataset in half and computing $f(x, y)_{i}$ for different subintervals within the two subsets. The difference, $\mathrm{d} f_{i}$, between the $f(x, y)_{i}$ is produced by comparison to a reference.

$$
\mathrm{d} f_{i}(x, y)=\frac{\left|f(x, y)_{i}-f(x, y)_{\text {ref }}\right|}{f(x, y)_{\text {ref }}}
$$

The mean, $\mu_{i}$, and standard deviation, $\sigma_{i}$, of the two dimensional $\mathrm{d} f_{\mathrm{i}}$ are taken as a measure of change between $f(x, y)_{i}$ and $f(x, y)_{\text {ref. }}$ The coefficient of variation, $V$, for the $\mu_{i}$ and $\sigma_{i}$,

$$
V_{\mu_{i}}=\sigma_{\mu_{i}} / \mu_{\mu_{i}}
$$

and,

$$
V_{\sigma_{i}}=\sigma_{\sigma_{i}} / \mu_{\sigma_{i}}
$$

represent the amount of variability among the $f(x, y)_{i}$. The coefficients of variation were computed for several different ensemble lengths for both flight data and random data. The results are shown in Fig 12(a). The flight data coefficients are from 5 to 10 times greater than those of the random data indicating increased variability in the flight data joint probability density function compared to the random data. This suggests that the flight data are less than strictly stationary.

Analyses which require stationarity may still be performed with some degree of confidence if the data can be shown to be weakly stationary. Bendat and Piersol [23] suggest a test for weak stationarity in which a set of statistics are taken from the data for several subintervals, as was done for the joint density function, above. The set are then tested for trends using a 'reverse arrangements' hypothesis test in which the number of transitions from 'less than' to 'greater than' are counted in the data. The results for the mean square and covariance of one channel of flight data are shown in Fig 12(b). Reversals were accumulated for 100 ensembles at each ensemble length. The bounds for the total number of reversals typical of

(a)

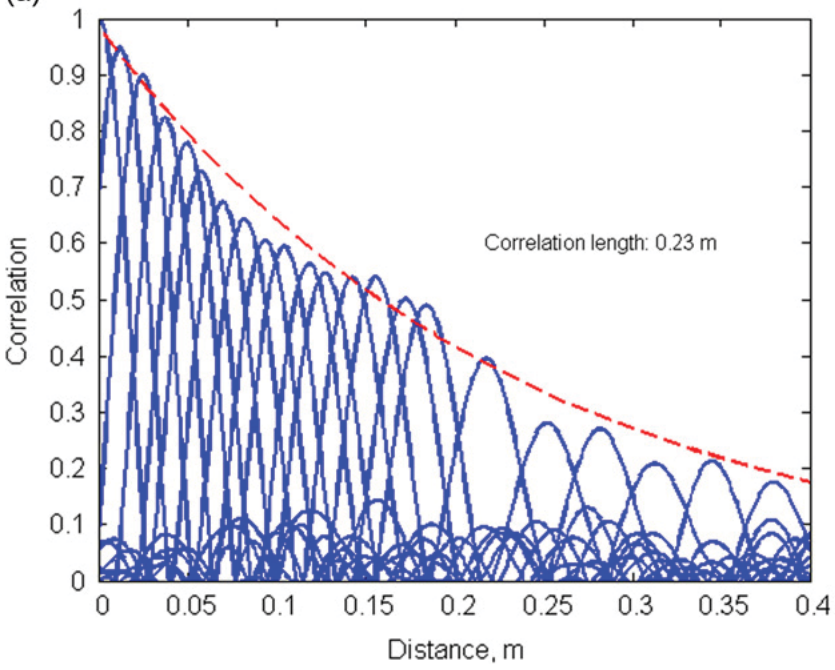

(b)

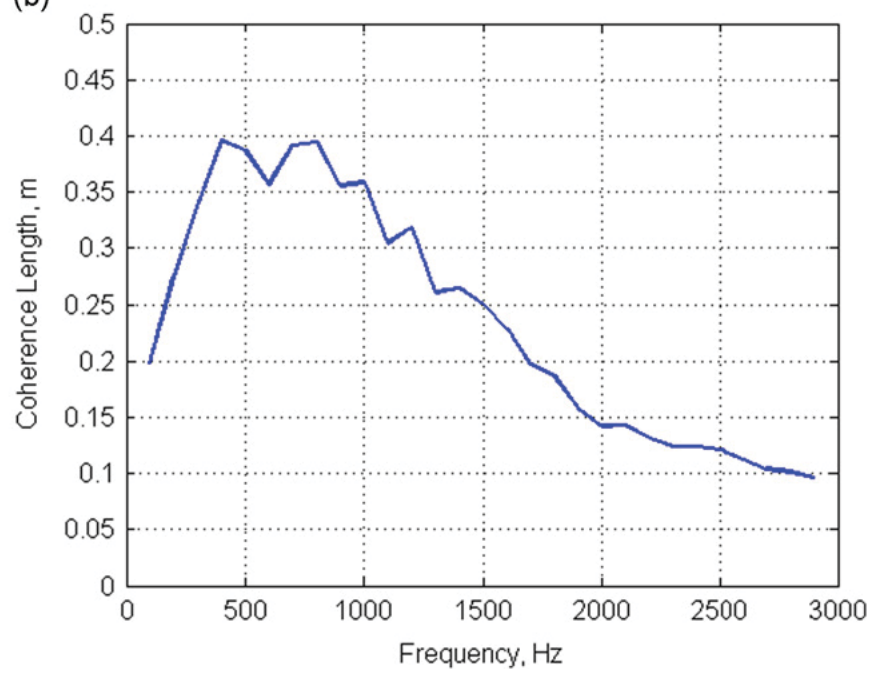

Fig 11. Correlation length (a), and coherence length (b), computed over a frequency band from $100 \mathrm{~Hz}$ to $3 \mathrm{kHz}$. 
(a)

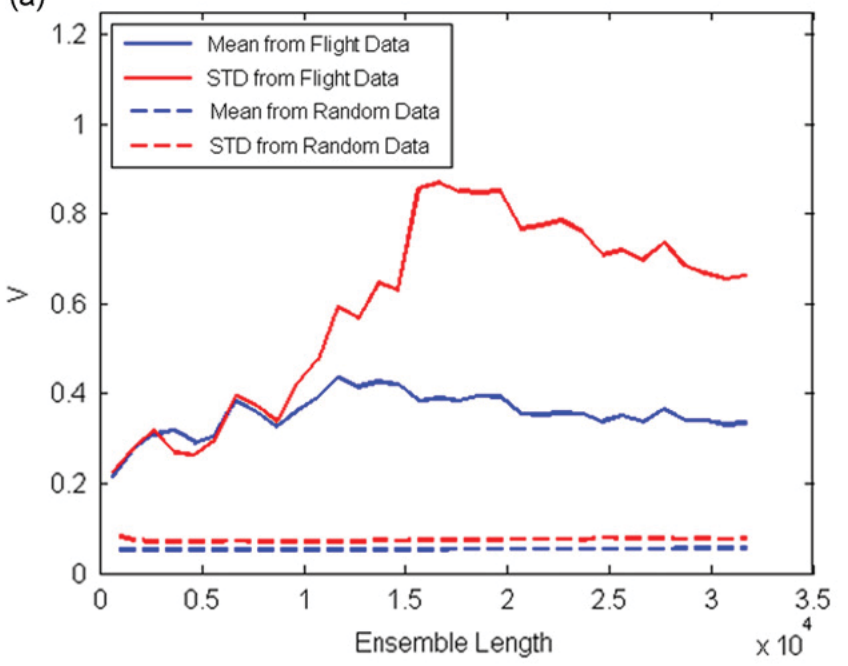

(b)

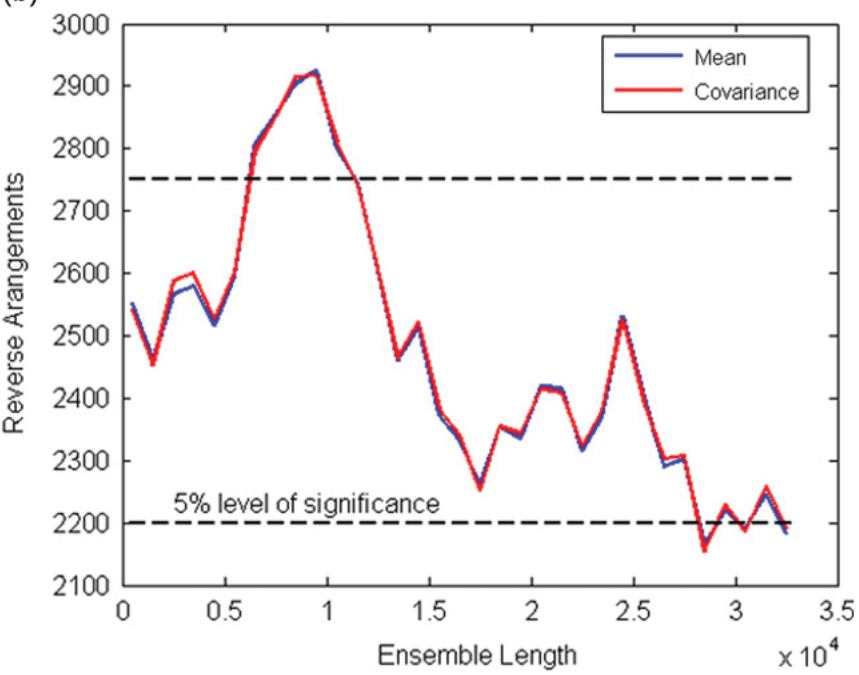

Fig 12. Coefficient of variation for change in $f(x, y)$ for flight data and random data (a), and test for weak stationarity using mean and covariance (b).

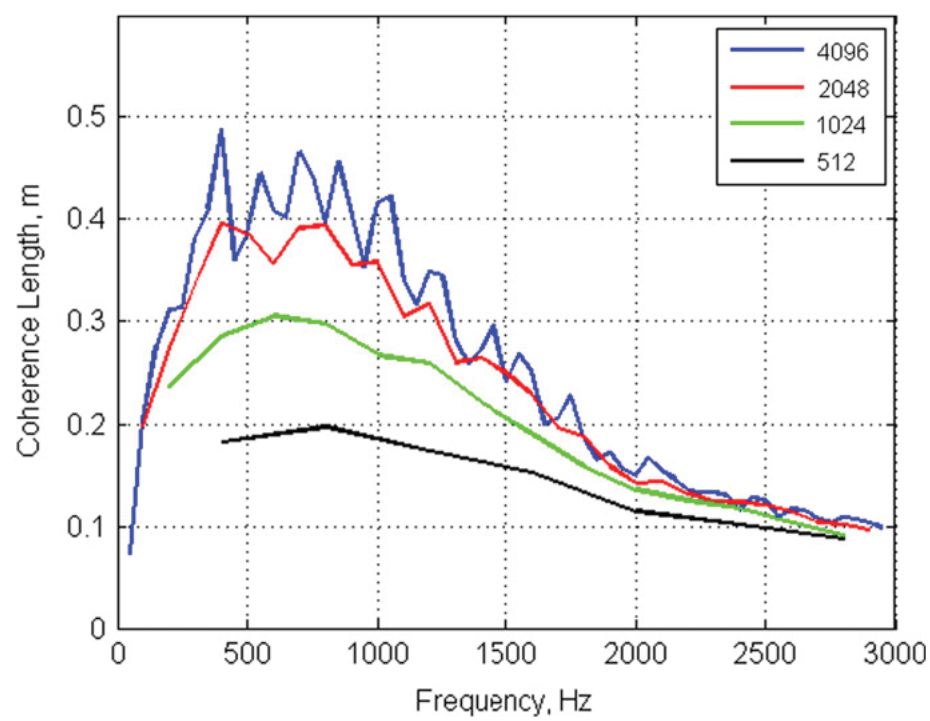

Fig 13. Coherence length computed at several ensemble lengths.

random data are given for a level of significance of 5\%. It can be seen that the measure is largely within the bounds except for the interval between 5000 and 10,000 samples. The data approaches the lower limit at 30,000 samples. These results indicate that, under most conditions the data are suited for averaged spectral analysis.

\subsection{Effects of convection}

The coherence length result shown in Fig 11(b) was computed with an ensemble length of 2048 samples resulting in a bin width of $100 \mathrm{~Hz}$. Unfortunately, the coherence length depends on ensemble length, converging with longer lengths. This is illustrated in Fig 13 where the coherence length was computed for different ensemble lengths and a constant 250 averages. The quality of fit of the exponential to the coherence decay is not altered as the ensemble length is varied and remains very good, e.g., see Fig 9(b). Normally the cross spectrum would be averaged to produce reliable statistics. In the case of turbulent flow, however, convection causes offset in the data contained in the ensembles, reducing coherence.

This is illustrated in Fig 14 where data from two sensors are shown and a prominent structure within the ensemble regions marked. The cross product between adjacent sensors would produce good results because the offset would be small. This would not be the case for a cross product between sensors located so far apart that the offset would introduce significant uncorrelated data. Fig 14(a) shows the structure highlighted for sensor 1 in ensemble 1 propagating to ensemble 2 for sensor $n$. The data in ensemble 1 , sensor 1 is, in this case, largely unrelated and, therefore, uncorrelated, to the data in ensemble 1 , sensor $n$, due to convection and not a decay in coherent power. 
(a)

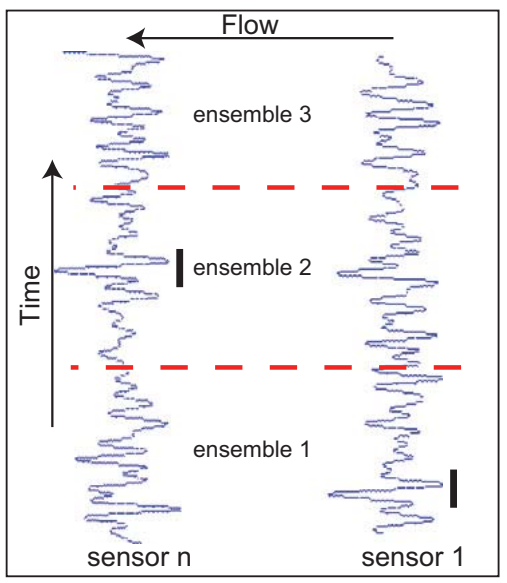

(b)

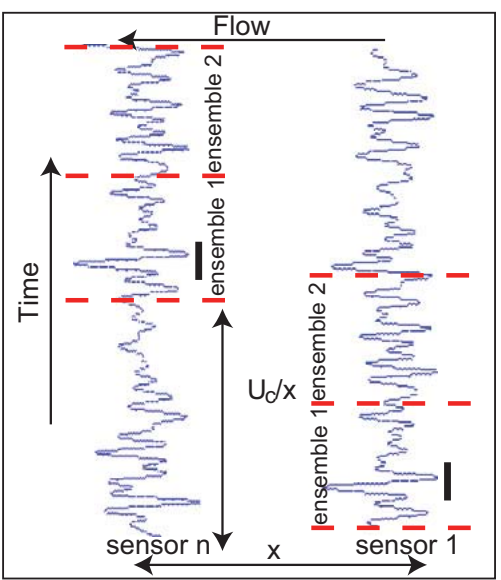

Fig 14. Illustration of ensemble averaging in flow, normal averaging (a), and convected averaging (b).

The reduction in $\gamma, \mathrm{d} \gamma$, caused by the convection can be estimated by

$$
\mathrm{d} \gamma=\frac{\Sigma_{i=1}^{\mathrm{d} N} d_{i}^{2}}{\Sigma_{i=1}^{N} d_{i}^{2}}
$$

where $N$ is the ensemble length, $d_{i}$ are the data and $\mathrm{d} N$ is the number of samples in the delay time created by the convection, Fig 14(b).

$$
\mathrm{d} N=\frac{x f_{s}}{U_{c}}
$$

where $x$ is the distance between the sensors, $f_{s}$ is the sample rate and $U_{c}$ the convection velocity. Using random data with a convection velocity of $150 \mathrm{~m} / \mathrm{s}$ and an ensemble length of 4096, the reduction in $\gamma$ is calculated to be 0.3 per meter of sensor separation. The reduction is greater for shorter ensembles and less for longer ensembles. This is precisely the behavior observed in Fig 13.

The effect of the flow can be reduced by shifting the ensemble boundaries according to the distance separating the sensors and the convection velocity, $U_{c}$, as shown in Fig 14(b). Now, related data is captured within the same ensemble buffer on each sensor. The measure of the mean convection velocity is not adversely affected and can be obtained using the common cross correlation or the cross spectrum to obtain the time or phase delay, respectively.

The convected cross correlation can be expressed as

$$
\chi\left(x_{0}, x_{1}, \tau, U_{c}\right)=\left\langle p\left(x_{0}, t_{0}\right) p\left(x_{1}, t_{0}+\tau+\frac{x_{1}-x_{0}}{U_{c}}\right)\right\rangle
$$

The convected cross spectrum is the Fourier transform of the convected cross correlation as before. The benefit of using the convected cross spectrum can be seen in Fig 15 where the coherence lengths now overlay each other with the exception of the $400 \mathrm{~Hz}$ case. The $400 \mathrm{~Hz}$ result is reduced due to averaging which occurs over the wide band of the bin width, much the same effect as was seen with the cross correlation.

\section{Data analysis}

The convected forms of the cross correlation and cross spectrum will now be used to analyse the flight data. The coherence length will be compared to historic values by comparison to Corcos and Efimtsov model parameters most often quoted in the literature. The convected cross correlation will be used to isolate coherent structures in the data and the nature of the correlation decay of the structures will be explored.

\subsection{Convected cross spectrum}

The Corcos model [6] for the cross spectrum assumes an exponential decay of correlated power with distance and can be written as

$$
\phi\left(r_{1}, r_{2}, \omega\right)=e^{-\alpha_{1} \omega\left|r_{1}\right| / U_{c}} e^{-\alpha_{2} \omega\left|r_{2}\right| / U_{c}} e^{i \omega r_{1} / U_{c}}
$$




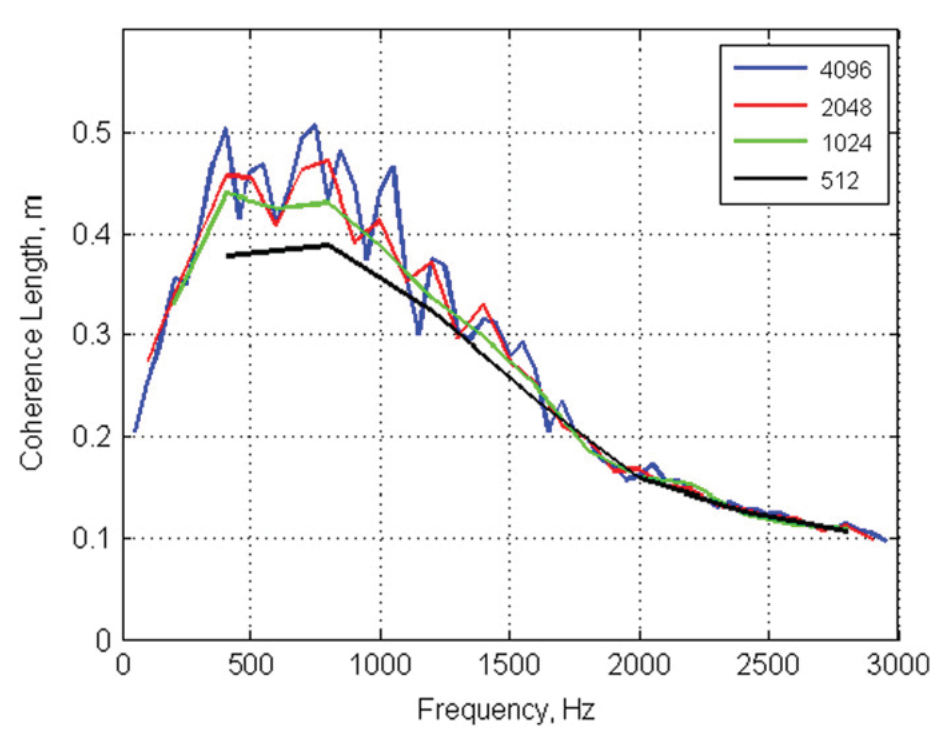

Fig 15. Coherence lengths computed using convected cross spectrum.

Table 2

Prevalent and derived coherence length parameters.

\begin{tabular}{|c|c|c|c|c|c|c|}
\hline & \multicolumn{3}{|c|}{ Streamwise } & \multicolumn{3}{|c|}{ Cross stream } \\
\hline & $a 1=\alpha 1$ & $a 2$ & $a 3$ & $a 1=\alpha 2$ & $a 2$ & $a 3$ \\
\hline Prevalent [1] & 0.1 & 72.8 & 1.54 & 0.77 & 548 & 13.5 \\
\hline $0.56 \mathrm{M}$ & 0.06 & 5.0 & 1.0 & 0.77 & 260 & 4.0 \\
\hline $0.7 \mathrm{M}$ & 0.06 & 5.0 & 1.0 & 0.77 & 260 & 4.0 \\
\hline $0.86 \mathrm{M}$ & 0.07 & 4.0 & 1.0 & 0.85 & 60 & 4.0 \\
\hline
\end{tabular}

Here $r_{1}, \alpha_{1}$ and $r_{2}, \alpha_{2}$ are the distance between two points and the decay constant for the streamwise and cross stream directions, respectively. The coherence length, $L_{\mathrm{coh}}$, is then

$$
L_{\mathrm{coh}, \mathrm{C}}=\frac{U_{c}}{\alpha \omega}
$$

The predicted coherence length is inversely proportional to frequency leading to over estimation of the value at low frequency when compared to actual data. Efimtsov [7] offered a correction for the coherence length as

$$
L_{\mathrm{coh}, E}=\delta\left[\left(\frac{a_{1} S h}{U_{c} / u_{\tau}}\right)^{2}+\frac{a_{2}^{2}}{S h^{2}+\left(a_{2} / a_{3}\right)^{2}}\right]^{-1 / 2}
$$

where $\delta$ is the boundary layer thickness, $S h=\omega \delta / u_{\tau}$ is the Strouhal number, $u_{\tau}$ is the friction velocity and parameters $a_{1 \ldots 3}$ are defined specifically for the streamwise and cross stream directions. The values of these parameters most often used in the literature are given in Table 2 along with values derived from the flight test data. The Efimtsov model is constrained by the Corcos model (through $a_{1}$ ) at mid to high frequencies. Parameter $a_{2}$ controls where the Efimtsov model breaks away from the Corcos curve. Parameter $a_{3}$ controls the low frequency roll off. The frequency and height of the peak in the coherence length curve can then be set by adjusting $a_{1}, a_{2}$ and $a_{3}$.

Fig 16(a) illustrates the fit of the Corcos and Efimtsov models to the $0.7 \mathrm{M}$ streamwise coherence length data. The fit is a compromise in that some error must be tolerated at the high frequencies to raise the curve to match the peak at lower frequencies. The changes in the parameters reflect an observed increase in coherence length. The original Efimtsov parameters predicted a maximum coherence length of $0.1 \mathrm{~m}$ at $1.5 \mathrm{kHz}$. Analysis of the flight data has the coherence length peaking at close to $0.5 \mathrm{~m}$ at $750 \mathrm{~Hz}$. Note that all flight conditions returned similar values for $a_{1}, a_{2}$ and $a_{3}$ indicating a consistent result. The cross stream cases were less well behaved, Fig 16(b). The Corcos parameter, $a_{1}$, was unchanged for the $0.56 \mathrm{M}$ and $0.7 \mathrm{M}$ cases and required a slight increase for the $0.86 \mathrm{M}$ case. The $a_{2}$ parameter had to be reduced by $50 \%$ for the lower speed cases and nearly $90 \%$ for the $0.86 \mathrm{M}$ case. The reduction in $a_{2}$ for the $0.86 \mathrm{M}$ cross stream case is of the same order as that required in $a_{2}$ for the streamwise case. The reason for this, as described earlier in section "Data Consistency" on page 9 , is that the 0.86 M cross stream spectrum maintained a pressure response indicative of outer layer coherent structure that diminishes at the slower speeds. 
(a)

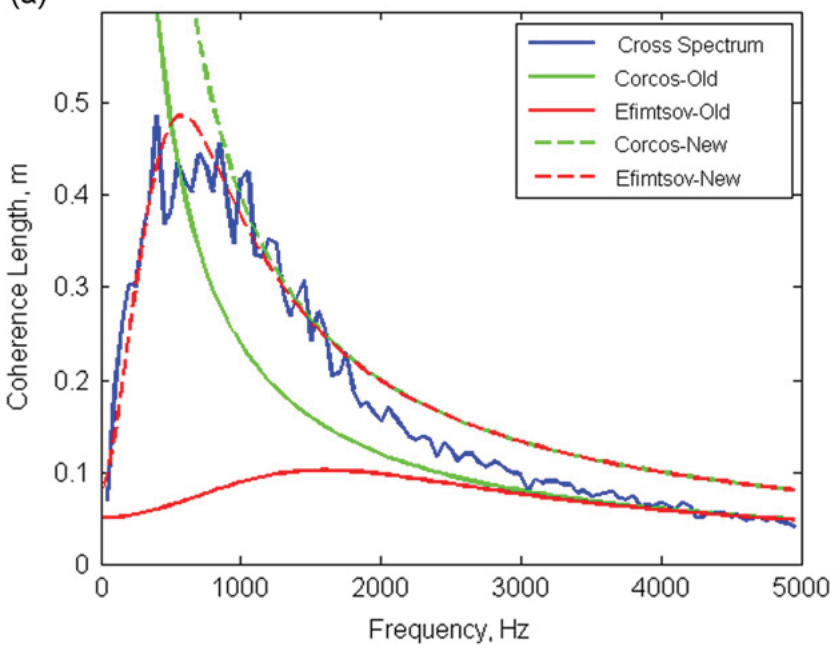

(b)

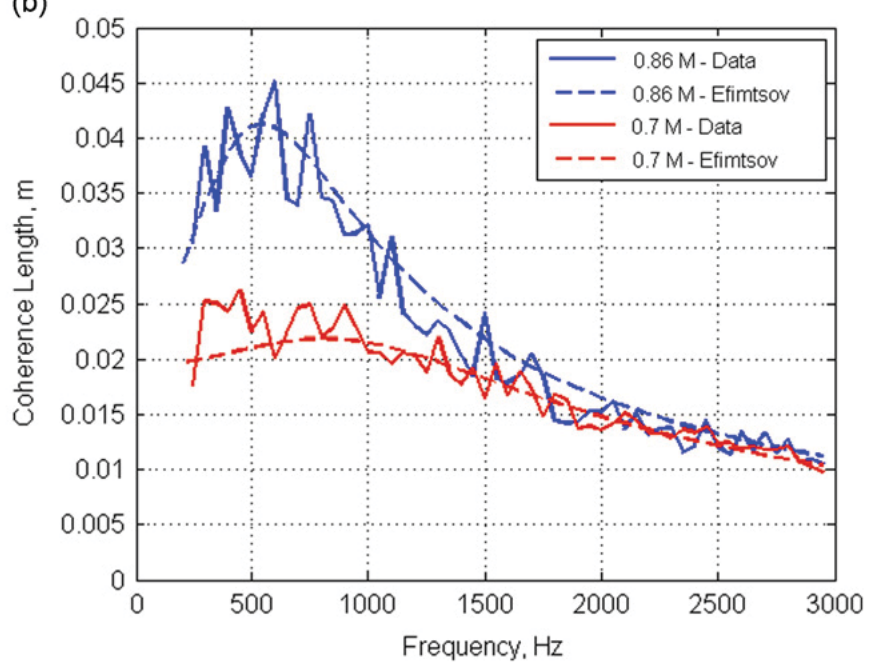

Fig 16. Coherence length dependence on frequency for $0.7 \mathrm{M}$ streamwise case (a), and comparison of $0.7 \mathrm{M}$ and $0.86 \mathrm{M}$ cross stream cases (b).

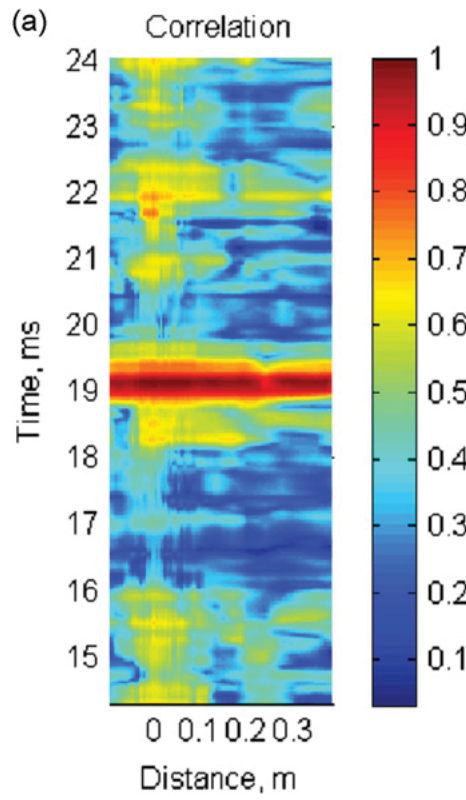

(b) Pressure, $\mathrm{Pa}$

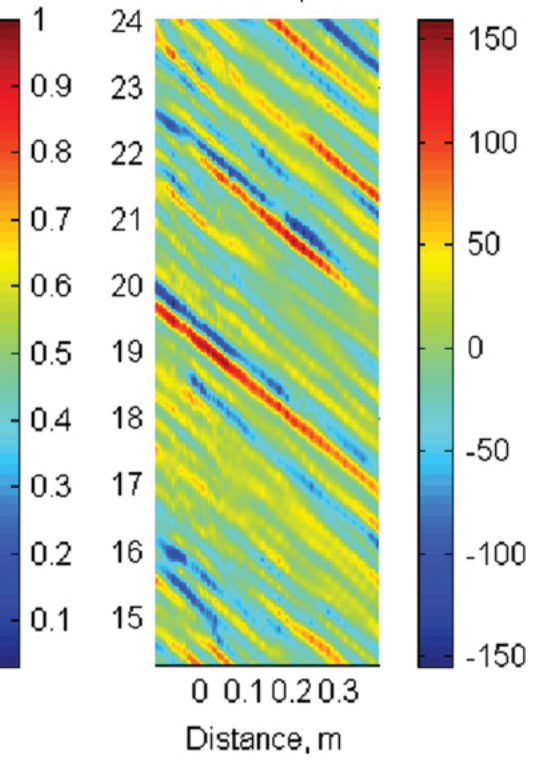

Fig 17. Convected cross correlation (a), and corresponding pressure (b).

\subsection{Detecting structure with the convected cross correlation}

The convected cross correlation is the basis of the convected cross spectrum so that the results presented in the previous section apply to the convected cross correlation as well. However, a form of the convected cross correlation can be used to detect and locate coherent structure in the wall pressure data so that these structures, which have been shown to have a large affect on the coherence length, can be studied. In this form of the analysis the number of samples, $N$, is reduced to an interval that will contain just 1 or 2 wavelengths of pressure data. To analyse broadband data the selected window size must be a compromise and was chosen to be 135 samples $(0.66 \mathrm{~ms})$ which corresponds to 1 wavelength at $1.5 \mathrm{kHz}$. The results of the convected cross correlation is scaled by the power over the array at the time the correlation is performed to enhance detection of the structure. The result is shown in Fig 17(a) alongside the corresponding pressure in (b). This particular interval of time is shown because it contained a clearly defined event in the correlation at 19 ms. Events of interest are chosen based on the peak value of the correlation for the reference sensor (located at distance $0 \mathrm{~m}$ ) and thus represent intervals of time when the power over the array is elevated. The significant event at $19 \mathrm{~ms}$ is seen to correspond to a long trail of both positive and negative pressure.

Once an event of interest is located, the nature of the phenomena can be explored. The evolution of the pressure waveform which created the dominant event shown in Fig 17 is shown in Fig 18(a). The event primarily consists of a 
(a)

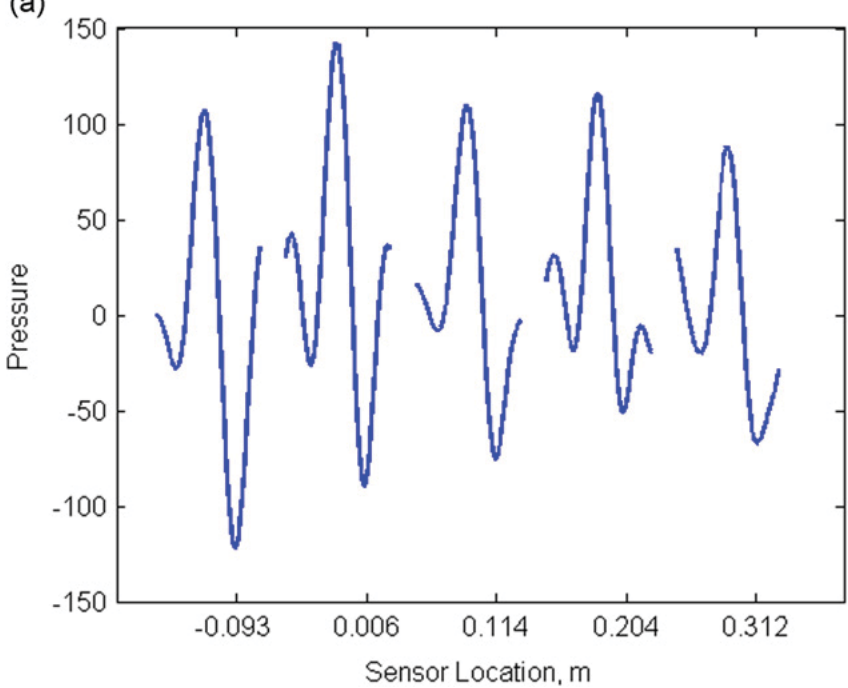

(b)

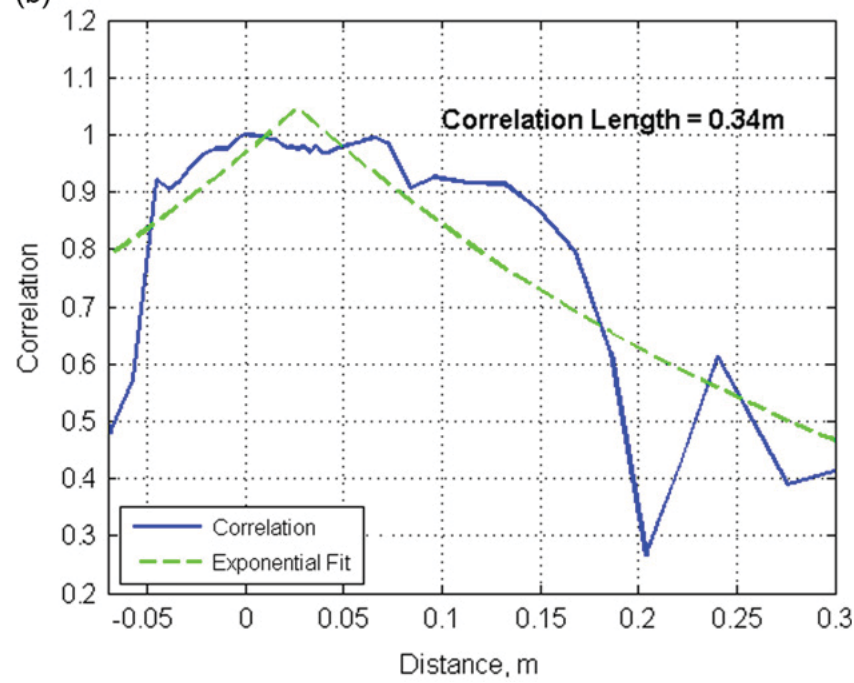

Fig 18. Pressure waveforms taken from 5 sensors around the time of the event (a), and typical correlation decay curve compared to exponential fit (b).

(a)

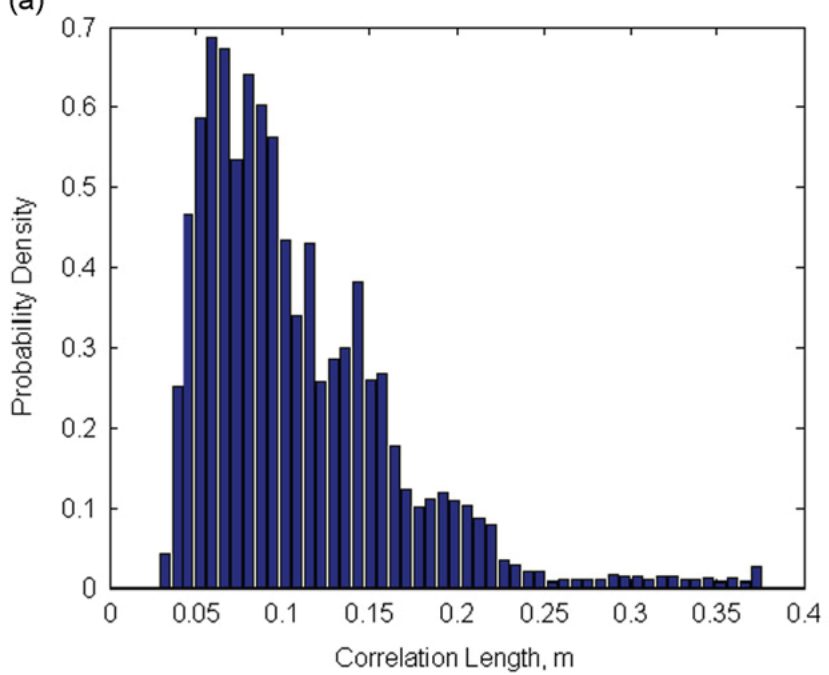

(b)

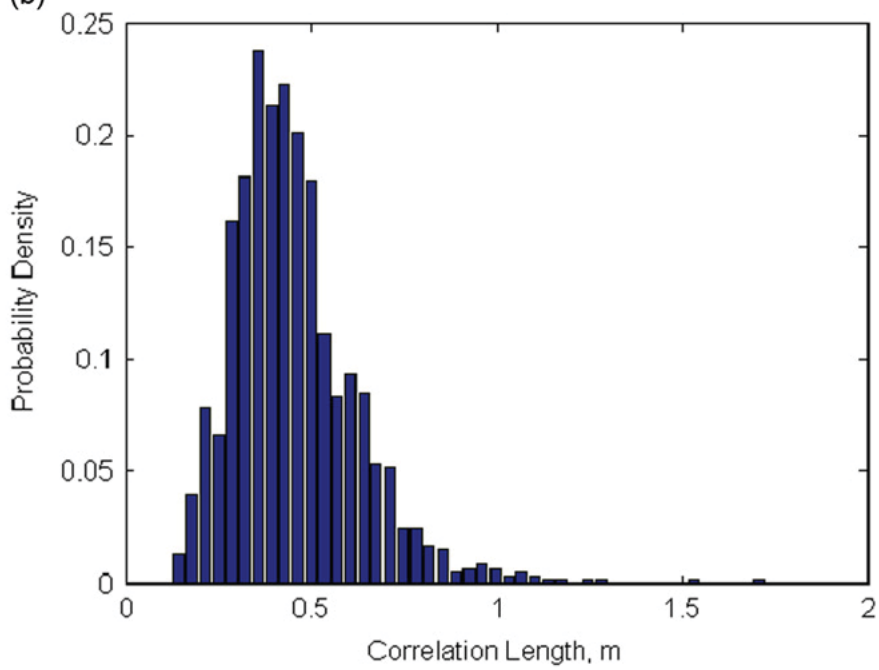

Fig 19. Distribution of correlation lengths, full frequency band (a), $1000 \mathrm{~Hz}$ (b). Exponential fit, red dashed line.

positive and negative peak. The shape and amplitude of the waveform is maintained across the array as might be expected considering the distance over which the correlation persists. This produces a relatively flat-topped correlation curve that does not resemble an exponential but is closer to what one might expect given Taylor's fixed field hypothesis [24]. An exponential fit on the correlation of this event produces an unrealistic correlation length of $8.6 \mathrm{~m}$. It is difficult to judge the quality of this estimate for this particular event because the event is so long, its beginning and end are not captured. A better understanding is obtained by considering a shorter event where the decay of the correlation can be observed, Fig 18(b). This event also has a relatively flat top which is seen to fall off rapidly on either side as has been found to be typical. An exponential fit to this curve returns a correlation length of $0.34 \mathrm{~m}$ which appears to over estimate the extent of the event. The exponential will return even more exaggerated correlation lengths for longer events due to the flatness of the correlation curve which is why the correlation length is calculated to be $8.6 \mathrm{~m}$ for the event in the previous example.

The lack of fit between the exponential curve and the correlation decay in the data seems to contradict the high quality of fit the exponential has for the correlation and coherence decay, Fig 9. This apparent discrepancy may be explained by considering the distribution of the instantaneous correlation lengths taken from data similar to that displayed in Fig 17(a). To compare the instantaneous correlation to the aggregate correlation in Fig 9(a), five thousand estimates of correlation lengths were taken from wideband data at each time step. The resulting distribution has the shape of a skew-normal distribution, Fig 19(a), with the distribution peaking at shorter lengths and then falling off towards longer lengths. When evaluating the aggregate correlation over the entire data block, the energy with the shorter correlation lengths will dissipate quickly while the energy with the longer correlations lengths will persist. This is what is observed in Fig 9(a) and what contributes to the correlation decay curve. When the frequency band is limited to $3 \mathrm{kHz}$, the data at a particular time 
appear as shown in Fig 18(a), so that it is possible to estimate the frequency of the structures as they occur. The correlation lengths of structures with frequencies of $1 \mathrm{kHz}$ were accumulated and are shown in Fig 19(b). It can be seen that the correlation length distribution of the narrow band data is similar in nature to the wideband data suggesting a similar mechanism at work when the coherence lengths are estimated. It may be possible then that, even though the correlation decay of the individual structures may not be best described as exponential, the aggregate behavior of all the events, as accumulated by the cross spectrum, produces a perceived exponential decay in coherence over distance.

\section{Discussion}

The power spectrum of the wall pressure of the flight data is governed by two distinct processes that have influence over two frequency ranges. The low to mid frequency bands exhibit behavior related to structures which exist in the log region of the boundary layer [18]. At higher frequencies the power spectrum becomes dominated by effects originating in the inner layer of the boundary layer. The two processes have quite different coherence length characteristics and create a lack of consistency across the frequency band which complicates attempts to model the coherence length with a single expression. Coherent structure with long correlation lengths appear to exist in the outer layer and influence the wall pressure mostly in the low to mid frequencies. The presence of the long life structures cause the wall pressure data to be non-homogeneous, that is, consisting of two or more processes.

The data exhibits characteristics similar to those found by Bhat [5] in that the coherence length increases from low frequency to a broad peak at mid frequencies, then falls off exponentially towards high frequencies. However, the measured coherence lengths appear to be much longer than those reported by Bhat ( $0.37 \mathrm{~m}$ vs. $0.13 \mathrm{~m})$ whose results are closer to those predicted by Efimtsov's model (Fig 16). While some of the observed disagreement may be attributed to the convective coherence losses described in this paper, it is believed that most are due to the high quality of the data obtained through the use of pinhole sensor ports in a single sheet of stainless steel. A previous analysis of flight data where great care was taken to achieve flush mounted sensors showed large errors in phase in the important low to mid frequency ranges [21]. These phase errors would have greatly reduced the measured coherence length between sensors. Similar estimates of long coherence lengths have been reported by at least one other recent study [15]. As mentioned above, the long coherence lengths are due primarily to the influence of coherent structure in the log region on the wall pressure. Cross stream measurements show that the influence of the coherent structure may vary depending on the position on the fuselage at which the measurements were made. Sensors located toward the windward side of the fuselage exhibited a peak in the power spectrum and elevated levels that were not observed in sensors located toward the leeward side of the fuselage. Peak levels were found to vary by as much as $5 \mathrm{~dB}$ over the $8 \mathrm{~cm}$ length of the array. The dependence of the auto and cross spectrum statistics on the position of the sensor makes generalizations of these statistics and the related correlation and coherence lengths more difficult.

The coherent power decay as derived from averaged cross spectrum statistics has been shown to be well represented by an exponential fit. This would lead one to expect that the coherent power in the log region structures that are responsible for a significant part of the measured coherence would also have an exponential decay. This has been shown not to be the case. The convected cross correlation has been used to detect the occurrence of the structures and evaluate their behavior. It has been observed that the detected events maintain a high, relatively constant correlation for most of the event life, falling off sharply at the ends so that the correlation curve resembles a rectangle function more than an exponential. To maintain consistency with prior work, the correlation lengths of the detected events were characterized by exponential fits. The distribution of these correlation lengths has been found to peak at short lengths and fall off at longer lengths. Thus, it is possible that the exponential nature of the coherence length obtained using the cross spectrum is not due to the shape of the coherent structures' correlation decay curves but a result of the distribution of the correlation lengths of these structures.

\section{Conclusions}

For the purpose of determining the parameters for models which estimate the wall pressure of the turbulent boundary layer over the surface of an aircraft fuselage, the current data are inadequate. Although it has been argued that the data are of high quality, the variation in pressure levels and spectra shape along the cross stream array leads one to conclude that a single set of parameters extracted from data taken at one location on the fuselage are insufficient. The hypothesis that fuselage curvature modulates the influence of log region structure on the wall pressure cannot be validated by these data alone. Given the available data, the Efimtsov model is able to adequately describe the streamwise coherence length frequency dependency although the result is a compromise between best fit for the lower versus the higher frequency ranges. To match observed coherence lengths that were almost five times longer than predicted, it was necessary to make considerable changes to the accepted Efimtsov parameters. It was not possible to define a single set of parameters which consistently predicted the cross stream coherence length for the three flight conditions tested.

The wall pressure of the turbulent boundary in this flight data is non-homogeneous and weakly stationary in space/ time. Care must then be taken when applying statistical analyses to wall pressure data such as this to avoid misleading results. Error due to convection may be compensated by using the convected forms of the cross correlation and spectrum. The different turbulent mechanisms that are at work in the inner and log regions are at the root of the inhomogeneous 
behavior and have greatest effect in different frequency bands resulting in a lack of consistency with frequency that may bias wide band analyses such as the cross correlation.

It is found that the nature of the correlation decay curve for the individual coherent structures is not well described by an exponential. While this seems, at first, to be at odds with the good fit between the coherence length obtained using the cross spectrum and the exponential, the distribution of correlation lengths for all the events is similar to a skew-normal distribution. It is conjectured that this characteristic of the flow is what is reflected in the cross spectrum statistics.

\section{Conflict of Interest}

The author confirms that there are no known conflicts of interest associated with this publication and there has been no significant financial support for this work that could have influenced its outcome.

\section{References}

[1] W.R. Graham, Boundary layer induced noise in aircraft, part I: the flat panel model, Journal of Sound and Vibration 192 (1) (1996) 101-120.

[2] C. Maury, P. Gardonio, S.J. Elliott, A wavenumber approach to modelling the response of a randomly excited panel, part II: application to aircraft panels excited by a turbulent boundary layer, Journal of Sound and Vibration 252 (1) (2002) 115-139.

[3] S.A. Hambric, Y.F. Hwang, W.K. Bonness, Vibrations of plates with clamped and free edges excited by low-speed turbulent boundary layer flow, Journal of Fluids and Structures 19 (2004) 93-110.

[4] M.K. Bull, Wall-pressure fluctuations beneath turbulent boundary layers: some reflections on forty years of research, Journal of Sound and Vibration 190 (3) (1996) 299-315.

[5] W.V. Bhat, Flight test measurement of exterior turbulent boundary layer pressure fluctuations on Boeing model 737 airplane, Journal of Sound and Vibration 14 (4) (1971) 439-457.

[6] G.M. Corcos, The structure of the turbulent pressure field in boundary-layer flows, Journal of Fluid Mechanics 18 (1963) $353-378$.

[7] B.M. Efimtsov, Characteristics of the field of turbulent wall pressure fluctuations at large reynolds numbers, Soviet Physics Acoustical 28 (4) (1982) 289-292.

[8] D.M. Chase, The character of the turbulent wall pressure spectrum at subconvective wavenumbers and a suggested comprehensive model, Journal of Sound and Vibration 112 (1) (1987) 125-147.

[9] G.M. Corcos, Resolution of pressure in turbulence, Journal of Acoustical Society of America 35 (2) (1963) $192-199$.

[10] M. Frigo, S.G. Johnson, FFTW: an adaptive software architecture for the FFT, Proceedings of the International Conference on Acoustics, Speech and Signal Processing 3 (1998) 1381-1384.

[11] W.R. Graham, A comparison of models for the wavenumber-frequency spectrum of turbulent boundary layer pressures, Journal of Sound and Vibration 206 (4) (1997) 541-565.

[12] Y.F. Hwang, W.K. Bonness, S.A. Hambric, Comparison of semi-empirical models for the turbulent boundary layer wall pressure spectra, Journal of Sound and Vibration 319 (2009) 199-217.

[13] S.A. Rizzi, R.G. Rackl, \& E.V. Andrianov, Flight test measurement from the TU-144LL structure/cabin noise experiment, NASA TM-2000-209858, 2000.

[14] B.M. Efimtsov, A.Y. Golubev, V.B. Kuznetsov, S.A. Rizzi, A.O. Andersson, R.G. Rackl, and E.V. Andrianov, Effect of transducer flushness on measured surface pressure fluctuations in flight, AIAA Paper 2005-0800, Jan. 2005.

[15] S. Finnveden, F. Birgersson, U. Ross, T. Kremer, A model of wall pressure correlation for prediction of turbulence-induced vibration, Journal of Fluids and Structures 20 (2005) 1127-1143.

[16] W.K. Blake, Mechanics of Flow-Induced Sound and Vibration, Vols. I and II, Academic Press, New York, 1986.

[17] T.M. Farabee, M.J. Casarelle, Special features of wall pressure fluctuations beneath turbulent boundary layers, Physics of Fluids A 3 (10) (1991) 2410-2420.

[18] N. Hutchins, Ivan Marusic, Evidence of very long meandering features in the logarithmic region of turbulent boundary layers, Journal of Fluid Mechanics 579 (2007) 1-28.

[19] D.J. Leclercq, X. Bohineust, Investigation and modelling of the wall pressure field beneath a turbulent boundary layer at low and medium frequencies, Journal of Sound and Vibration 257 (3) (2002) 477-501.

[20] G.c. Canavos, Applied Probability and Statistical Methods, Little, Brown and Co., Boston, 1984.

[21] D. Palumbo, Deriving lifetime maps of coherent structure in the turbulent boundary layer, AIAA Journal 46 (4) (2008) $810-823$.

[22] D. Palumbo, Persistent structure in the turbulent boundary layer, 11th AIAA/CEAS Aeroacoustics Conference, Monterey, CA, May 23-25, 2005, AIAA2005-2854.

[23] J.S. Bendat, A.G. Piersol, Random Data, John Wiley \& Sons, Inc., New York, 2000.

[24] G.I. Taylor, The spectrum of turbulence, Proceedings of the Royal Society 164 (1938) 476. 